\title{
Competition between drift and spatial defects leads to oscillatory and excitable dynamics of dissipative solitons
}

\author{
P. Parra-Rivas, ${ }^{1,2}$ D. Gomila, ${ }^{2}$ M. A. Matías, ${ }^{2}$ P. Colet,${ }^{2}$ and L. Gelens ${ }^{1,3,4, *}$ \\ ${ }^{1}$ Applied Physics Research Group (APHY), Vrije Universiteit Brussel (VUB), 1050 Brussels Belgium \\ ${ }^{2}$ Instituto de Física Interdisciplinar y Sistemas Complejos, IFISC (CSIC-UIB), Campus Universitat de les Illes Balears, \\ E-07122 Palma de Mallorca, Spain \\ ${ }^{3}$ Laboratory of Dynamics in Biological Systems, KU Leuven Department of Cellular and Molecular Medicine, \\ University of Leuven, B-3000 Leuven, Belgium \\ ${ }^{4}$ Department of Chemical and Systems Biology, Stanford University School of Medicine, Stanford, California 94305-5174, USA
}

(Received 6 July 2015; published 20 January 2016)

\begin{abstract}
We have reported in Phys. Rev. Lett. 110, 064103 (2013) that in systems which otherwise do not show oscillatory dynamics, the interplay between pinning to a defect and pulling by drift allows the system to exhibit excitability and oscillations. Here we build on this work and present a detailed bifurcation analysis of the various dynamical instabilities that result from the competition between a pulling force generated by the drift and a pinning of the solitons to spatial defects. We show that oscillatory and excitable dynamics of dissipative solitons find their origin in multiple codimension-2 bifurcation points. Moreover, we demonstrate that the mechanisms leading to these dynamical regimes are generic for any system admitting dissipative solitons.
\end{abstract}

DOI: 10.1103/PhysRevE.93.012211

\section{INTRODUCTION}

Dissipative solitons (DSs) are exponentially localized structures appearing in dissipative systems far from thermodynamic equilibrium [1]. DSs are formed due to the balance between nonlinearity and spatial coupling and then driving and dissipation. They can be found in a large variety of fields such as chemistry [2], gas discharges [3] and fluid mechanics [4], vegetation and plant ecology [5], and optics [6,7].

DSs can undergo instabilities leading to a wide variety of temporal dynamics, such as periodic oscillations [8-11] and chaos [11-13]. It has also been theoretically shown that in nonlinear Kerr cavities, DSs can display excitable behavior despite the fact that the local dynamics (namely the dynamics in the absence of spatial coupling) are not excitable [14]. This differs from systems where excitability is a local property, such as neural models [15], because excitability is now an emergent property of the DSs. However, while DS excitability may be useful in information processing [16], in two-dimensional (2D) spatial systems oscillatory and excitable DSs are elusive and for most systems only static DSs have been reported. This situation contrasts with one-dimensional (1D) systems such as fiber cavities, where periodic oscillating DSs have been demonstrated experimentally and theoretically $[11,13]$.

We recently showed that the competition between spatial inhomogeneities and drift provides a way to induce oscillations and excitability of DSs [17]. This competition introduces an oscillatory instability, which can lead to a regime in which DSs are pulled one by one from the defect (referred to as a train of DSs) and to an excitable regime in which the DSs stay pinned in the defect but can be pulled out by a transient perturbation to the system.

Here we provide a detailed analysis of the dynamical regimes induced by the competition between drift and defects first reported in Ref. [17]. For convenience we will consider the 1D Swift-Hohenberg equation (SHE). Contrary to the SHE

\footnotetext{
*lendert.gelens@kuleuven.be
}

for a complex field which shows a rich variety of dynamical behaviors as illustrated in Refs. [18,19], the SHE for a real field can be considered as a prototypical system that does not exhibit any time-oscillatory dynamics. Based on the results obtained in the SHE model for a real field we will argue that oscillatory and excitable dynamics are a general feature for any system admitting DSs in the presence of drift and defects. Such inhomogeneities or defects are unavoidable in any experimental setup, and drift is also often present in many optical, fluid, and chemical systems. In optical systems this can be caused by misalignments of the mirrors [20,21], nonlinear crystal birefringence [22], or parameter gradients [23], while in fluid and chemical systems drift is due to fluid flow [24,25].

In Sec. II, we will first introduce the SHE for a real field, a generic amplitude equation describing pattern formation in a large variety of systems [26,27]. We then introduce additional terms in the SHE such that it accounts for both drift and spatial inhomogeneities. Next, we proceed to analyze the effect of the individual and combined effect of drift and defects by exploring bifurcation diagrams in function of the strength of the drift and defect term (Secs. III A-III D). This analysis will reveal various regions of dynamical behavior, where we will highlight the distinctive properties of oscillatory (Sec. IV) and excitable (Sec. V) DS dynamics. These dynamics are then shown to unfold from two Takens-Bogdanov (TB) $[28,29]$ codimension-2 bifurcation points in Sec. VI. In Sec. VII, we discuss the generality of this drift-defect mechanism (i) by considering a different parameter region of the SHE and (ii) by assuming the spatial inhomogeneity is in the gain in the SHE. Finally, we end by discussing the general aspects of the work and its particular relevance in optics (Sec. IX).

\section{THE SWIFT-HOHENBERG EQUATION}

The SHE for a real field $u$ in one spatial dimension $x$ can be written as follows:

$$
\partial_{t} u=-\left(\partial_{x}^{2}+k_{0}^{2}\right)^{2} u+r u+a u^{2}-g u^{3},
$$


with $g>0$ to avoid divergences. Of particular interest for our purposes is that the SHE is variational (for periodic boundary conditions) or, in other words, the dynamics follows a gradient [26,30]. This implies that the right-hand side of Eq. (1) can be written as the (functional) derivative of a certain functional, namely

$$
F=\int_{0}^{L} d x\left\{-\frac{1}{2} r u^{2}+\frac{1}{2}\left[\left(\partial_{x}^{2}+k_{0}^{2}\right) u\right]^{2}-\frac{1}{3} a u^{3}+\frac{1}{4} g u^{4}\right\} .
$$

The dynamics in the SHE are such that $F$ decreases in time until it reaches a local minimum, i.e., a steady state that minimizes $F$. As a consequence, the SHE cannot exhibit dynamical regimes that differ from stationary states (i.e., this excludes oscillations and chaos). The simplicity and lack of any oscillatory dynamics will allow us to clearly identify how drift and defect terms adjust the dynamical behavior of DSs.

The homogeneous steady states (HSSs) of the SHE are

$$
\begin{gathered}
u_{s}=0, \\
u_{s}=\frac{1}{2 g}\left[a \pm \sqrt{a^{2}+4 g\left(r-k_{0}^{4}\right)}\right] .
\end{gathered}
$$

The linear stability of the HSSs in response to finite wavelength perturbations,

$$
u(x, t)=u_{s}+\epsilon \exp (\Gamma t+i k x),
$$

is determined by the dispersion relation

$$
\Gamma(k)=r+2 a u_{s}-3 g u_{s}^{2}-\left(k_{0}^{2}-k^{2}\right)^{2} .
$$

The HSSs undergo a generalized Turing (or modulational) instability [26,27] as a function of $r$ if $\Gamma(k)=\Gamma^{\prime}(k)=0$ [a double zero of $\Gamma(k)$ ] for a given critical value $k=k_{c}$. In other words, the HSS is Turing unstable when $\Gamma(k)$ develops a maximum for some $k=k_{c}$ and this maximum is exactly zero. The trivial solution $u=0$ becomes Turing unstable first at $r=0$ for perturbations with critical wave number $k_{c}= \pm k_{0}$. At $r=k_{0}^{4}$ this trivial state is also unstable to uniform perturbations (perturbations with critical wave number $k_{c}=0$ ). When $a>\sqrt{27 / 38} g$ (see Ref. [31]) a spatially periodic solution emerges subcritically from the Turing instability at $r=0$. Together with this subcritical pattern, two sets of DSs, one with an even number of peaks and one with an odd number of peaks, unfold from the $r=0$, forming what is known as a homoclinic snaking structure [31]. Unless mentioned otherwise, we will consider $g=1, a=1.2$, and $k_{0}^{2}=0.5$ throughout this work, ensuring the presence of a subcritical pattern.

Following Refs. [32-34], Fig. 1 shows the homoclinic snaking of the branch with an odd number of peaks. The different branches of steady-state solutions have been found solving

$$
0=-\left(\frac{d^{2}}{d x^{2}}+k_{0}^{2}\right)^{2} u+r u+a u^{2}-g u^{3},
$$

using a Newton-Raphson method that allows finding both stable and unstable solutions. The solutions are plotted in the $L_{2}$ norm, $\|u\|^{2}:=\int(u(x))^{2} d x$. The different branches corresponding to DS solutions oscillate back and forth around

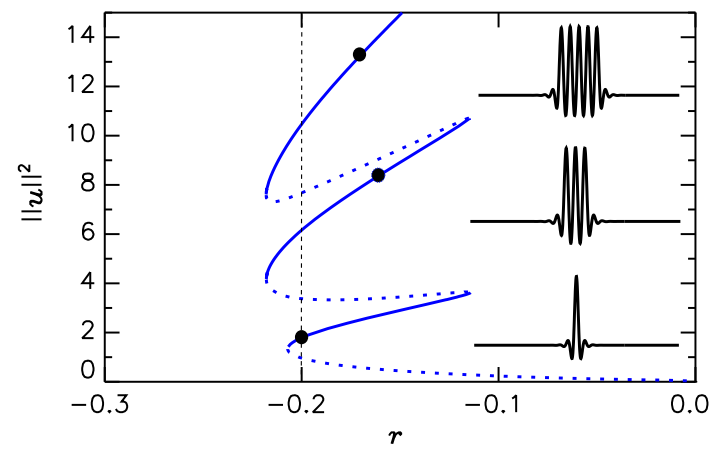

FIG. 1. Homoclinic snaking for $k_{0}^{2}=0.5, a=1.2$, and $g=1$. The solid (dashed) lines represent stable (unstable) DSs. The vertical dashed line shows the value of $r$ chosen in the analysis in Sec. III. The insets show the spatial profile of the DSs with an odd number of peaks, corresponding to the solid black circle on each stable branch.

the Maxwell point (the location where the potential $F$ is equal for the subcritical pattern and the trivial solution), explaining the term "snaking structure." The solid lines represent the stable solutions and the dashed lines the unstable ones. After crossing each saddle-node bifurcation [30] at the right-hand side of the diagram the DSs add a pair of peaks symmetrically at both sides of the existing peaks. Some examples of such DS solutions are shown in the insets. In the next sections, we will simplify our analysis by fixing the value of the control parameter $r$ to -0.2 and by focusing on the dynamical properties of a DS consisting of a single peak.

\section{OVERVIEW OF THE DRIFT-DEFECT INDUCED DYNAMICS IN THE SWIFT-HOHENBERG EQUATION}

\section{A. A Swift-Hohenberg equation with drift and defect}

In this paper a drift is modeled in the SHE by introducing a gradient term

$$
c \partial_{x} u
$$

with $c$ the group velocity. The spatial defect is defined by a single Gaussian profile $b(x)$ of height $h$ and half-width $\sigma$ that is located in the center $x_{0}$ of a system with domain width $L$ $\left(x_{0}=L / 2\right)$ :

$$
b(x)=h \exp \left[-\left(\frac{x-x_{0}}{\sigma}\right)^{2}\right] .
$$

The SHE modified to include drift and defect thus reads:

$$
\partial_{t} u=r(x) u+a u^{2}-g u^{3}-\left(\partial_{x}^{2}+k_{0}^{2}\right)^{2} u-c \partial_{x} u+b(x) .
$$

To avoid drifting DSs from re-entering the domain on the opposite side, we use a super-Gaussian gain profile $r(x)$,

$$
r(x)=r_{0}-1+\exp \left[-\left(\frac{x-x_{0}}{\Lambda}\right)^{18}\right] .
$$

In this way the system has an effective width $2 \Lambda$, and all drifting DSs disappear at the boundaries. Unless mentioned otherwise, we choose $\sigma=2.045$ (roughly half the width of a DS), $\Lambda=94.0842$, and $r_{0}=-0.2$. 


\section{B. Dissipative solitons in the presence of drift}

For periodic boundary conditions, in the presence of drift, but without defects $(h=0, c \neq 0)$, spatial reversibility under the transformation $(u, x, t) \rightarrow(u,-x, t)$ is broken and the system can display convective instabilities. The solutions of Eq. (10) are steadily drifting asymmetric states [35]. By introducing a change of variables $(x, t) \rightarrow(x-c t, t)$ this drift can be removed and the system can be studied in a reference frame moving at velocity $c$. In that frame the solutions can be only stationary and the system dynamics is still variational.

\section{Dissipative solitons in the presence of a defect}

In the presence of defects, but without drift $(c=0, h \neq 0)$, Eq. (10) is still variational with a Lyapunov functional given by

$$
F_{h}=F+\int_{0}^{L} b(x) u(x) d x,
$$

and, as before, only steady-state solutions of Eq. (10) exist.

One of the main consequences of the defect term is the breaking the invariance of Eq. (1) under the translational transformation $x \mapsto x+a$, with $a \in \mathbb{R}$. The steady-state solutions are now pinned at the location of the spatial defect. We can gain a better understanding of the effects of such spatial inhomogeneities by looking at the bifurcation diagram showed in Fig. 2, generated for periodic boundary conditions. In this diagram we plot $\|u\|_{\text {sup }}:=\max [u(x)]$ as a function of the control parameter $h .\|u\|_{\text {sup }}$ is chosen here instead of the $L_{2}$ norm because it allows all different branches to be more clearly visible in this case. Each branch corresponds to a different type of solution. Examples of each type of solution are shown in
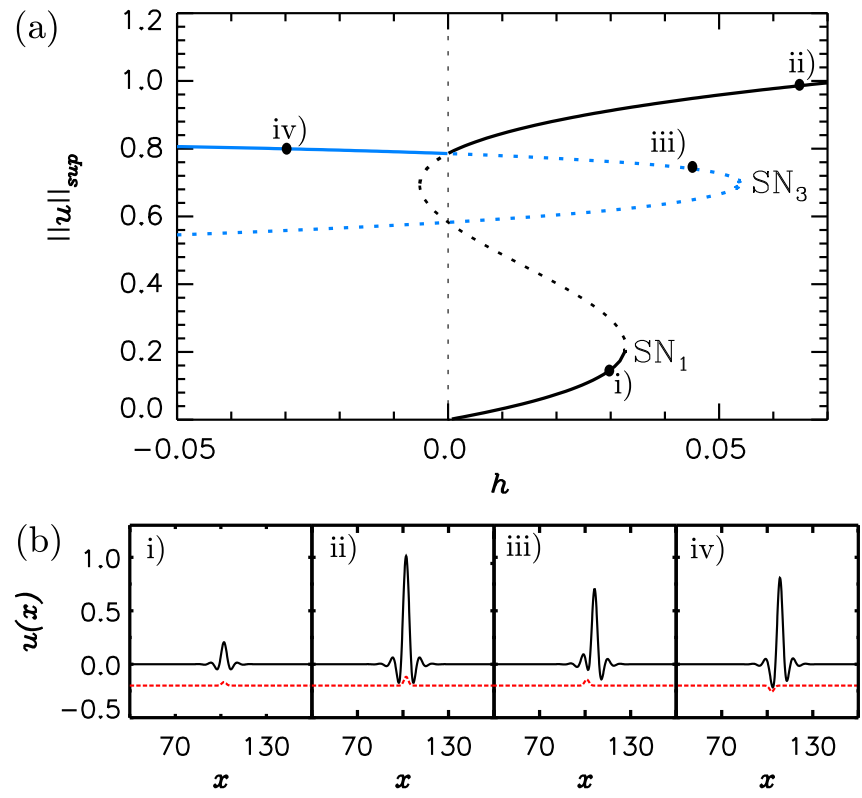

FIG. 2. (a) Bifurcation diagram (maximum $\|u\|_{\text {sup }}$ ) as a function of the strength of the spatial defect $h$ for $c=0$. (b) Examples of the steady-state solutions (black solid lines) corresponding to the labeled branches, together with the profiles of the spatial defect (red dashed lines). $\mathrm{SN}_{1}$ and $\mathrm{SN}_{3}$ represent saddle-node bifurcations. the bottom panels. The stable steady-state solutions (i), (ii), and (iv) are the main attractors of the system. Solution (i) corresponds to the fundamental solution, a small deformation of the trivial solution. Solutions (ii) and (iv) correspond to a large amplitude DS pinned at its center or at the first oscillation of its tail, respectively. Finally, solution (iii) also represents a large-amplitude DS pinned at the first minimum of its oscillatory tail, but the DS is unstable.

Due to the breaking of the translational symmetry, a transcritical bifurcation [30] takes place at $h=0$. In this bifurcation branch (ii) becomes unstable, while branch (iii) is stabilized. Physically, at $h=0$ the defect goes from being a bump to a hole. DSs tend to sit at the maximum of any inhomogeneity, such that DSs centered at the hole become unstable and shift their position until the hole coincides with the first minimum of its tail. Branch (iii) corresponds to pinned DSs whose maximum is at the right of the defect. There also exists a degenerate branch with the maximum at the left side of the defect. This degeneracy is broken by drift as discussed in the next section. In the remainder of the study, we will focus on the DSs whose maxima are located downstream of the defect, since this branch will reconnect to branch (ii) in the presence of drift.

\section{Dynamics in the presence of drift and defect}

When considering the joint effect of drift and defect, it is no longer possible to describe the system in a moving reference frame and the system no longer follows gradient dynamics minimizing a functional. As a result, steady-state solutions can undergo instabilities leading to time oscillatory dynamics. As previously mentioned, in the presence of only drift ( $h=$ $0, c \neq 0)$, spatial reversibility is broken and as result solutions are asymmetric and move with a constant velocity. By also introducing a spatial inhomogeneity $(h \neq 0, c \neq 0)$, the drift can be compensated for if the pinning force due to the defect is stronger than the drift force trying to pull it out. As we will show below, this competition between pinning (defect) and depinning (drift) can give rise to a wide range of dynamical instabilities.

In Fig. 3(a) we show how the bifurcation diagram of Fig. 2 is modified when introducing a small drift $(c=0.0015)$. Even a small drift is enough to break the spatial symmetry and leads to the appearance of a pair of imperfect transcritical bifurcations [30] that split the solution branches at $h=0$. A saddle-node on the invariant circle (SNIC) bifurcation [30] now connects branch (ii) (stable large amplitude DS) to branch (iii) (unstable large amplitude DSs pinned at the first tail oscillation). Likewise, the branch (iv) (stable large amplitude DSs pinned at the first tail oscillation) connects to the unstable middle branch DS, which previously (for $c=0$ ) connected branch (i) and (ii). A new saddle-node bifurcation $\mathrm{SN}_{2}$ arises from the middle branch, while the saddle-node bifurcations $\mathrm{SN}_{1}$ and $\mathrm{SN}_{3}$ were already present for $c=0$. Examples of the different profiles in the presence of a defect and small drift are shown in Fig. 3(b). In what follows, we will focus on the region in parameter space where $h>0$.

Figure 4 shows how this bifurcation structure (for $h>0$ ) is modified as the drift speed $c$ is gradually increased. Figure 4(a) shows the single snakelike branch for a weak breaking of 
(a)
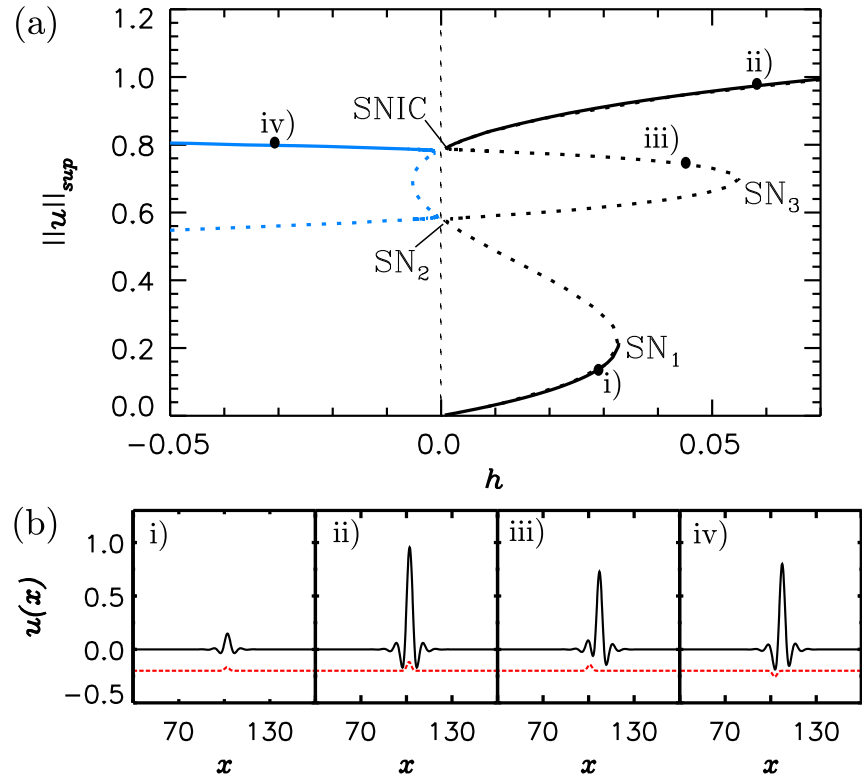

FIG. 3. (a) Bifurcation diagram (maximum $\|u\|_{\text {sup }}$ ) as a function of the strength of the spatial defect $h$ for $c=0.0015$. (b) Examples of the steady-state solutions (black solid lines) corresponding to the labeled branches, together with the profiles of the spatial defect (red dashed lines).

the reflection symmetry $(c=0.05)$. The crosses indicate the extrema of oscillations in the amplitude of the DS at the central defect location. These oscillations originate at the SNIC and terminate at a fold-of-cycles (FC) bifurcation [30], which will be discussed in more detailed later.

As the symmetry is progressively broken (increasing values of $c$ ) the branches stretch [Fig. 4(b)] and $\mathrm{SN}_{1}$ coalesces with $\mathrm{SN}_{2}$ in a codimension-two point known as a hysteresis or cusp bifurcation [30] that we denote as $\mathrm{C}_{1}$, which takes place at $c_{\mathrm{C}_{1}}=0.11772$. This process is illustrated in more detail in the two parameter ( $c$ and $h$ ) phase diagram shown in Fig. 5. For values of $c$ just below $c_{\mathrm{C}_{1}}$, a Hopf bifurcation [30] appears subcritically [Fig. 4(b)]. We denote this subcritical bifurcation as $\mathrm{H}^{+}$, where the symbol + points to the subcriticallity of the bifurcation.

As $c$ is increased further, the SNIC turns into a saddle-node bifurcation $\mathrm{SN}_{4}$ through another codimension-two point, referred to as a saddle-node separatrix-loop (SNSL) bifurcation $[28,29]$. At $c_{\mathrm{C}_{2}}=0.1642, \mathrm{SN}_{4}$, and $\mathrm{SN}_{3}$ coalesce in another cusp bifurcation that now we label as $\mathrm{C}_{2}$, see Fig. 5. In Fig. 4(c) we show the bifurcation structure structure for $c$ just below $c_{\mathrm{C}_{2}}$. In the inset one can see that another Hopf bifurcation $\mathrm{H}^{-}$has also appeared. In this case, the Hopf is supercritical (hence the symbol -).

Finally, for larger values of $c$, there is a single monotonic branch of steady-state solutions [Fig. 4(d)]. From the supercritical Hopf $\mathrm{H}^{-}$a stable limit cycle appears, which persists until it destabilizes at $\mathrm{FC}$, and disappears in $\mathrm{H}^{+}$.

Overall the phase diagram shown in Fig. 5 has five main dynamical regions, which are labeled A-E in Figs. 4-6:

Region A: The fundamental solution is stable. The system can display Type II excitability for parameters close to the FC line (see more details in Sec. V).

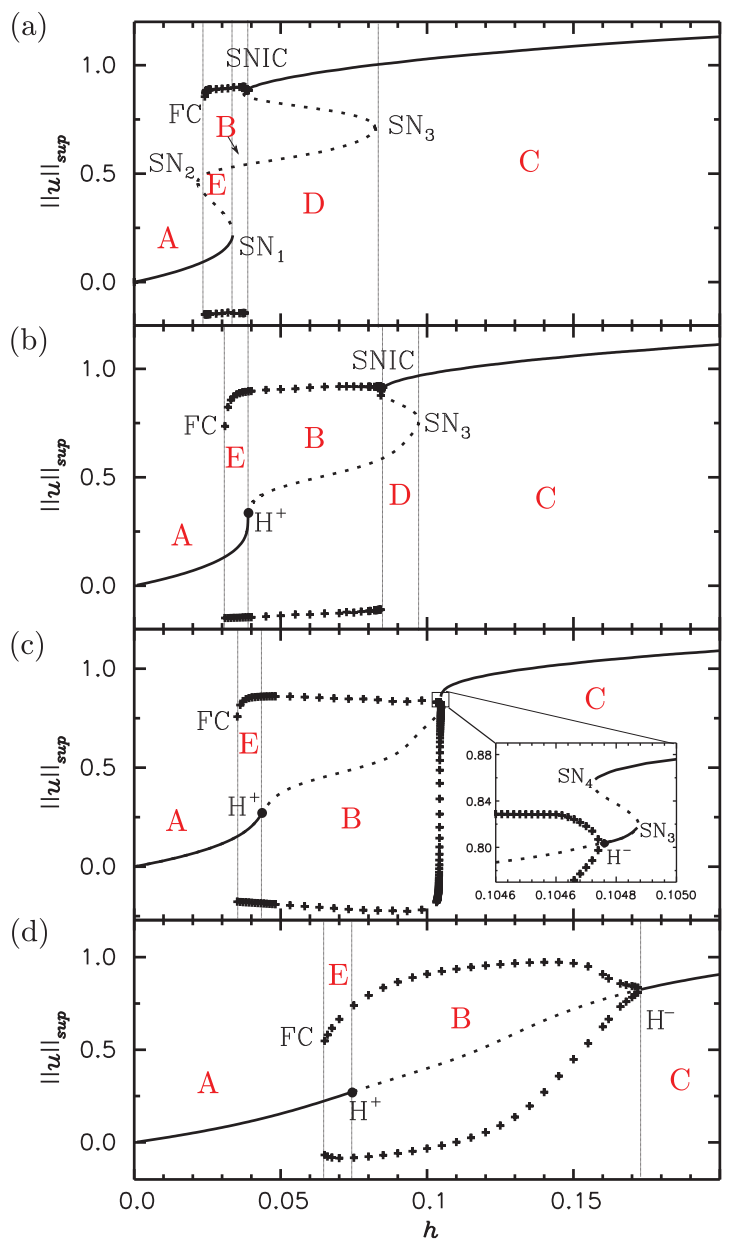

FIG. 4. Bifurcation diagram (maximum $\|u\|_{\text {sup }}$ ) as a function of the strength of the spatial defect $h$ for (a) $c=0.05$, (b) $c=0.12$, (c) $c=0.162$, and (d) $c=0.4$. Crosses indicate the extrema (maxima and minima) of the DS oscillatory amplitude. The main dynamical regions A-E are labeled in red.

Region B: DSs oscillate periodically in time (see more details in Sec. IV).

Region C: Stable large amplitude DSs pinned at the defect exist, while Type II excitability occurs for parameters close to the $\mathrm{H}^{-}$line (see more details in Sec. V).

Region D: Stable large amplitude DSs pinned at the defect exist, while the system can also admit Type I excitability (see more details in Sec. V).

Region E: The fundamental solution and oscillatory DSs coexist.

Figure 6 shows a zoom of the two-parameter phase diagram shown in Fig. 5. The zoom allows to better observe the overlap that exists between regions $\mathrm{A}$ and $\mathrm{D}$ (bounded by the SNIC and $\mathrm{SN}_{1}$ ) and between regions $\mathrm{A}$ and $\mathrm{E}$ (bounded by $\mathrm{SN}_{1}, \mathrm{SN}_{2}$, and FC).

In the next sections, we will discuss in more detail the properties of the oscillatory (Sec. IV) and excitable (Sec. V) dynamical regions, and we will show how the different bifurcations unfold from two Takens-Bogdanov codimensiontwo points (Sec. VI). 


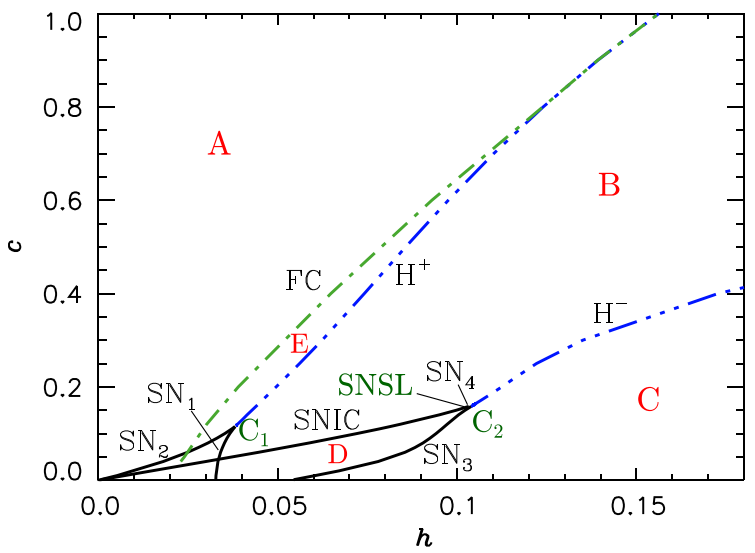

FIG. 5. Two-parameter ( $c$ vs $h$ ) phase diagram of the system for $a=1.2$ and $r=-0.2$. The bifurcation lines and regions A-E are explained in the main text.

\section{OSCILLATORY DYNAMICS}

In regions $\mathrm{B}$ and $\mathrm{E}$, static DSs are unstable. This instability leads to DSs that remain pinned at the defect but whose amplitude oscillates periodically in time. In Fig. 4, crosses indicate the maximum and minimum amplitude of the oscillatory DS pinned at the defect. In Fig. 4(b), where $c=0.12$, stable oscillations originate from the SNIC and disappear at the FC. For $c=0.12$ and $h=0.04$, Fig. 7(a) shows how the time-periodic oscillations of the pinned DS affect the spatial dynamics in the whole domain. The oscillations correspond to the periodic creation of a DS at the defect that then drifts away, thus generating a train of DSs originating from the defect. These drifting DSs disappear at the boundary of the domain due to the absorbing boundary conditions that are used, i.e., the super-Gaussian gain coefficient $r(x)$ given by (11). For all numerical simulations involving drifting structures we have used such absorbing boundary conditions, while periodic boundary conditions have been used to determine the bifurcation diagrams. Since the absorbing boundaries are located far away, close to the pinning defect the profile of the localized solutions is very similar for both boundary conditions. Furthermore, the instabilities of these localized solutions are associated to modes whose spatial profiles are also localized and are thus practically independent of

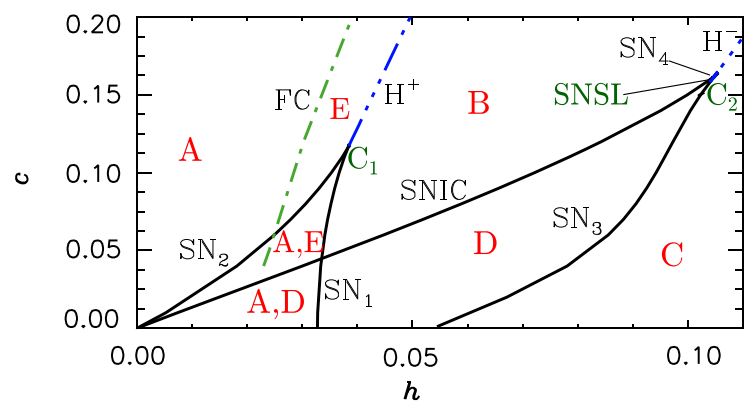

FIG. 6. Zoom of the two-parameter phase diagram shown in Fig. 5, such that the overlap between regions A and D, as well as $\mathrm{A}$ and $\mathrm{E}$ is visible. $a=1.2$ and $r=-0.2$. (a)

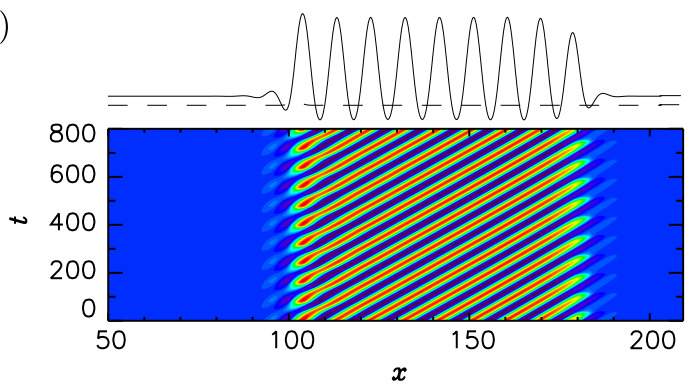

(b)

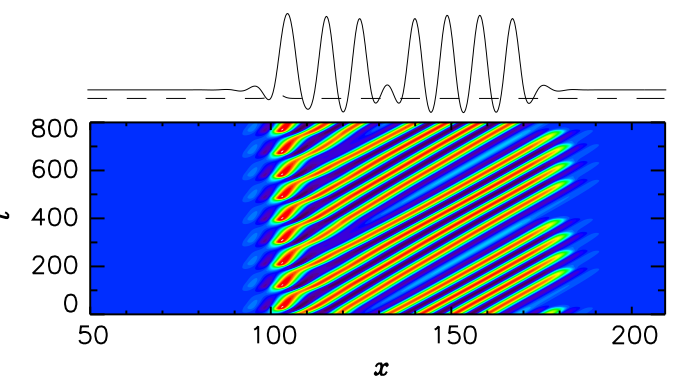

(c)

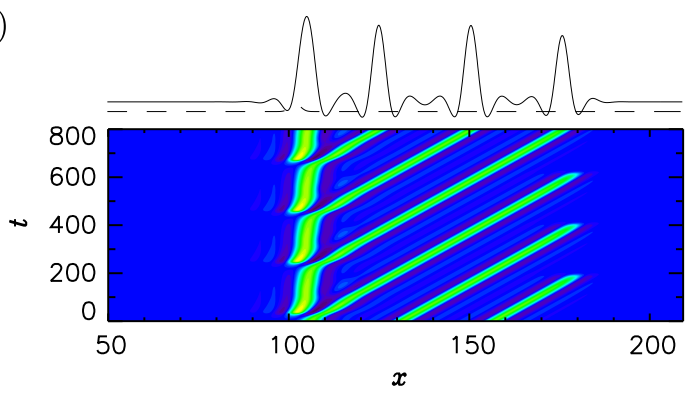

FIG. 7. Contour plots of $u(x, t)$ showing the spatiotemporal evolution of a train of DSs for $h=0.04$ (a), $h=0.06$ (b), and $h=0.08$ (c) for $c=0.12$ and $L=209$. Above the contour plots the spatial profile $u(x, t=800)$ is plotted.

the boundary conditions. As a consequence, the observed instabilities are the same for both kinds of boundary conditions.

When increasing $h$ further $(h=0.06)$, the oscillations start showing a second time scale [Fig. 7(b)], which becomes more pronounced for even large $h=0.08$ [Fig. 7(c)]. Such oscillations with two time scales are characteristic of systems displaying relaxation oscillations, as, for instance, described by the Van der Pol equation [28]. Figure 8(a) shows in more detail the time evolution of the amplitude of the DS in the center of the domain $x_{0}=L / 2$, confirming the presence of fast and slow time scales. The fast time scale corresponds to the nucleation process in which a DS is created at the inhomogeneity, while the slow one is the time it takes to detach a DS once it is formed due to the drift. The time to nucleate a DS is basically independent of the drift strength, while the escape time strongly depends on it. Figure 8(b) shows snapshots of the full spatial profile during one period of such an oscillation.

For larger values of $c$, two types of oscillations are observed [see Fig. 4(d) for $c=0.4$ ]. First, for $h<0.158$, the regular train of solitons is found as shown in Fig. 9(a). Second, for larger values of $h$, close to the supercritical Hopf bifurcation $\mathrm{H}^{-}$, the strength of the defect is large enough to prevent the advection of the DS but nevertheless the competition between drift and pinning at the defect induces small amplitude 

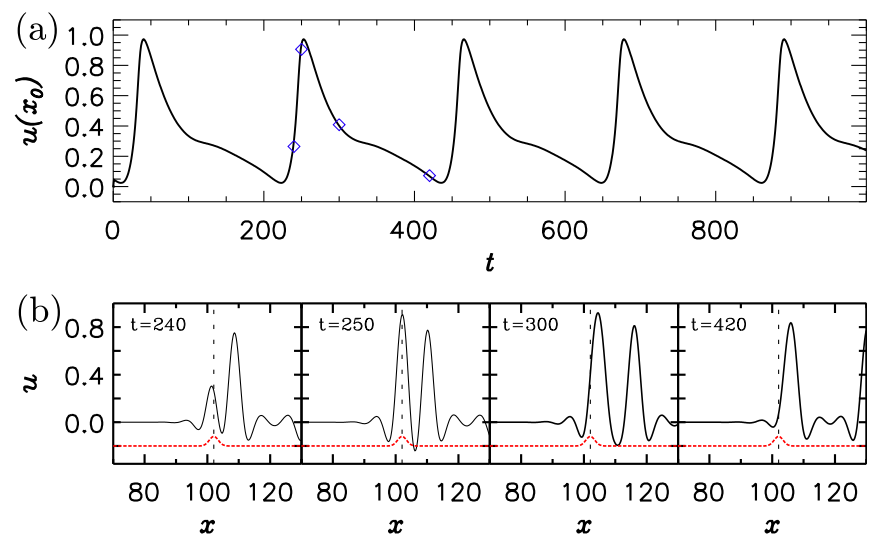

FIG. 8. (a) Time evolution of the magnitude $u\left(x_{0}\right)$ at the center of the domain $\left(x_{0}=L / 2\right)$ for a train of DSs. $h=0.08, c=0.12$, and $L=209$. (b) Snapshots of the spatial profile $u(x, t)$ at different times [as indicated in individual panels and corresponding to the blue diamonds in panel (a)], illustrating the growth and depinning of a DS from the spatial defect. The defect is shown in a red dashed line.

(a)

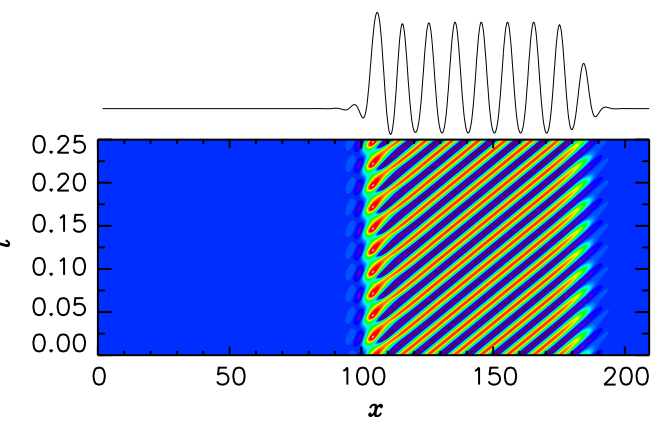

(b)
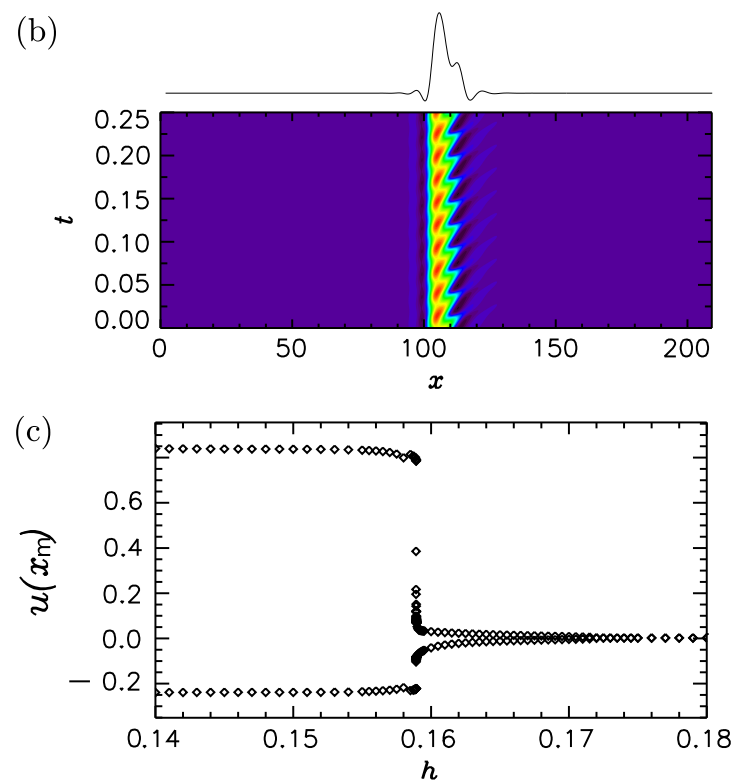

FIG. 9. Contour plots of $u(x, t)$ showing the spatiotemporal evolution of a train of DSs for $h=0.136$ (a) and a small amplitude oscillation for $h=0.164$ (b). $c=0.12$ and $L=209$. Above the contour plots the spatial profile $u(x, t=0.25)$ is plotted. (c) Canard explosion illustrated by plotting the maxima and minima of an oscillating DS, evaluated at $u\left(x=x_{m}\right)$ as function of $h$, for $x_{m} \approx 123$ and $c=0.4$. oscillations of the DS [see Fig. 9(b)]. The transition between both types of oscillatory regimes occurs very suddenly through what it is called a canard explosion. One refers to a canard explosion whenever varying a parameter (here $h$ ) leads to a very fast transition from small amplitude limit cycles to large amplitude relaxation oscillation cycles. This very fast transition happens within an exponentially small range of the control parameter. In Fig. 9(c) the extrema of $u\left(x_{m}\right)$ are plotted, where $x_{m} \approx 123$ was chosen to be in the tail of the DS such that one can clearly differentiate between the small oscillations of the DS and the train of DSs. The canard explosion occurs for $h \approx 0.158$ close to $\mathrm{H}^{-}$. This limit cycle corresponding to the train of DSs originating at the canard location remains stable all the way to a FC bifurcation, where it becomes unstable and disappears in $\mathrm{H}^{+}$.

In the next section, we will show that close to these oscillatory regions there also exist different types of excitability of DSs.

\section{DISSIPATIVE SOLITON EXCITABILITY}

A system is said to be excitable if perturbations below a certain threshold decay exponentially, while perturbations above this threshold induce a large response before going back to the resting state. Having a threshold is thus one of the basic ingredients in order to have excitability. A second ingredient is a reinjection mechanism in the phase space that forces the system to go back to the resting state. Here that reinjection mechanism corresponds to the combination of drift and absorbing boundary conditions such that any DS is eventually removed from the domain. The time-scale separation present in the relaxation oscillations (train of DSs) is a strong indication of excitability: The fast time scale is related with the triggering of the perturbation, and the slow time scale is related with the time required for the system to go back to the resting state (see Fig. 9).

In the literature it is common to find a classification for excitability that is based on whether or not there is a divergence of the period of the limit cycle involved in the dynamics [15]. Excitability is defined to be of Type I if the period diverges close to the bifurcations involved in the creation or destruction of the limit cycle, while it is of Type II if the period does not diverge and remains almost constant when approaching the bifurcation. Before discussing our system in more detail, we will briefly discuss these two types of excitability.

Type I excitability is related with two specific bifurcations, a saddle loop (SL) bifurcation (also referred to as a homoclinic bifurcation) [30] or a SNIC bifurcation. In both bifurcations, the stable manifold of a saddle point plays the role of a separatrix in phase space, so only perturbations bringing the system beyond this threshold trigger an excitable excursion [16]. A clear signature of this excitability is the divergence of the period of the oscillations that appear or disappear with each bifurcation. As we mentioned before, in a SNIC, a saddle-node bifurcation occurs inside a limit cycle. Before the SNIC, stable manifolds of the saddle and node states transverse to the center manifold are organized by an unstable focus inside a limit cycle. At one side of the bifurcation the system exhibits oscillatory behavior, while at the other side the dynamics of the system is excitable. Although this bifurcation 


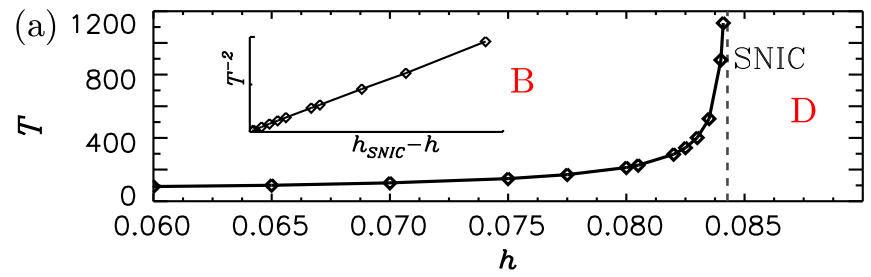

(b)
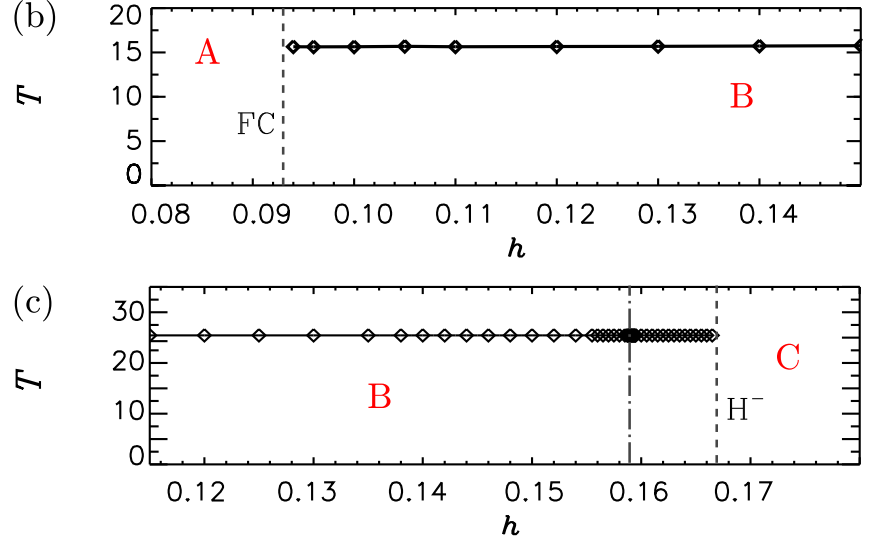

FIG. 10. Scaling of the oscillation period $T$ in function of $h$ for Type I (a) and Type II [(b) and (c)] excitability. (a) $c=0.12$; (b) $c=0.6$; (c) $c=0.4$.

is local in (one-dimensional) flows on the circle, it has global characteristics in higher-dimensional dynamical systems, so it is also termed a local-global bifurcation. The best way of characterizing the presence of that bifurcation is analyzing how the period of the cycle behaves close to it. For the SNIC it is known that the period of the cycle diverges as one approaches the bifurcation [see Fig. 10(a)], following the power law

$$
T \propto\left(h-h_{\mathrm{SNIC}}\right)^{-1 / 2} .
$$

Another kind of Type I excitability is mediated by a SL bifurcation [14] in which a stable limit cycle collides with a saddle and disappears. Here the unstable manifold of the saddle plays the role of sepatatrix in phase space. In this case the period diverges logarithmically when approaching the bifurcation,

$$
T \propto \ln \left(h-h_{\mathrm{SL}}\right) .
$$

One of the main differences between those two mechanisms of Type I excitability is that the SNIC mediated one is easier to observe than the SL one. Due to the square-root scaling law, it occurs over a broader parameter range.

Type II excitability is related with the presence of a subcritical Hopf bifurcation $\mathrm{H}^{+}$or a supercritical Hopf $\mathrm{H}^{-}$ bifurcation with a canard explosion. In this case there is no saddle involved, and, as a consequence, there is no separatrix in phase space. For this reason the threshold is not very well defined and one refers to a quasithreshold [15]. In Type II excitability the period of the oscillations remains practically constant as one approaches the point where such oscillations are created or destroyed [see Figs. 10(b) and 10(c)].

In this work, excitability can be found in regions $\mathrm{A}, \mathrm{C}$, and $\mathrm{D}$ in Fig. 5. In order to induce an excitable excursion, we use a suitable transient parameter change instead of perturbing the state of the system [16]. The transient parameter change should bring the system into the oscillatory region B. It should be long enough so the system starts to oscillate following the periodic limit cycle but shorter than the period of the limit cycle. Proceeding in this way, a pulse can be excited before returning to the resting state. Here we will use perturbations in $h$ modifying the defect strength for a short time. We redefine the profile $b(x, t)$ as follows to incorporate this transient perturbation:

$$
b(x, t)=\left[h+\Delta h H\left(t, t_{0}, t_{1}\right)\right] \exp \left[-\left(\frac{x-x_{0}}{\sigma}\right)^{2}\right],
$$

with the step function $H\left(t, t_{0}, t_{1}\right)$ defined as

$$
H\left(t, t_{0}, t_{1}\right)= \begin{cases}0, & \text { if } t<t_{0} \\ 1, & \text { if } t_{0}<t<t_{1} . \\ 0, & \text { if } t>t_{1}\end{cases}
$$

Here, without loss of generality, we have chosen $t_{0}$ as the time at which the perturbation starts and $\Delta t=t_{1}-t_{0}$ (with $\left.t_{1}>t_{0}\right)$ as the duration of the perturbation.

\section{A. Type I excitability (SNIC): Region D}

In our system, Type I excitability mediated by the SNIC bifurcation can be found in region D, close to the SNIC line separating regions B and D (Figs. 5 and 6). For steady states with parameters in region $\mathrm{D}$, a perturbation that brings the system beyond the excitability threshold triggers the unpinning of a DS leading to an excitability excursion. This excursion is shown in Fig. 11. The initial state corresponds to the parameters $c=0.12$ and $h=0.085$. By applying a perturbation $\Delta h=-0.035$ in $h$ for a time period $\Delta t=10$, the system is brought into region $B$ for a time that is insufficient to complete a full limit cycle. The perturbation is long enough, however, to allow a DS to be pulled out of the defect and drift out of the finite-size domain, bringing the system back to its initial state. The divergence of the period of oscillations in region B close to the SNIC can be seen in Fig. 10(a), confirming the presence of Type I excitability.

\section{B. Type II excitability $\left(\mathrm{H}^{+}\right.$and $\left.\mathrm{H}^{-}\right)$: Regions $\mathrm{A}$ and $\mathrm{C}$}

Type II excitability is found in region A, close to regions B and $\mathrm{E}$, and it is mediated by the subcritical Hopf bifurcation $\mathrm{H}^{+}$(Figs. 5 and 6). An example of a typical excursion is shown in Fig. 12. The initial and final resting state is not a high-amplitude DS but a low bump solution, the fundamental solution. A perturbation of this fundamental solution in region A beyond the excitability threshold (bringing the system into region B) allows the system to generate a DS, which then drifts away, resetting the system to its resting state. If one applies a perturbation such that the system crosses $\mathrm{FC}$, but not $\mathrm{H}^{+}$, then the system will find itself in region $\mathrm{E}$ which is bistable. The best way to trigger an excitable excursion is to perturb the system in the region where $\mathrm{FC}$ and $\mathrm{H}^{+}$are almost tangent. This way the perturbation required to excite an excursions will be smaller. Actually, the system is excitable even for $h=0$, although, in this case, very large perturbations are required to induce an excursion.

The second region of Type II excitability is found in region $\mathrm{C}$, again close to region $\mathrm{B}$, but this time it is mediated by the 
(a)

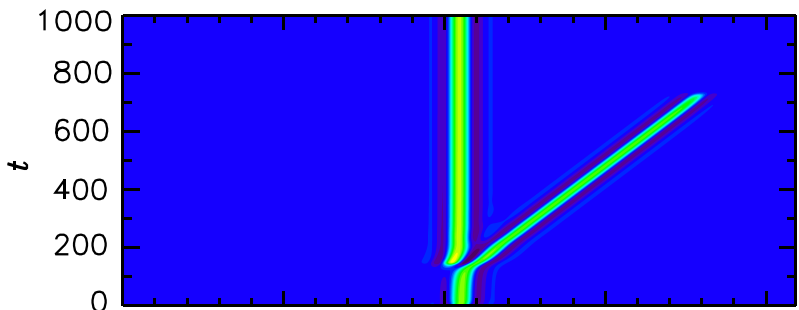

(b)

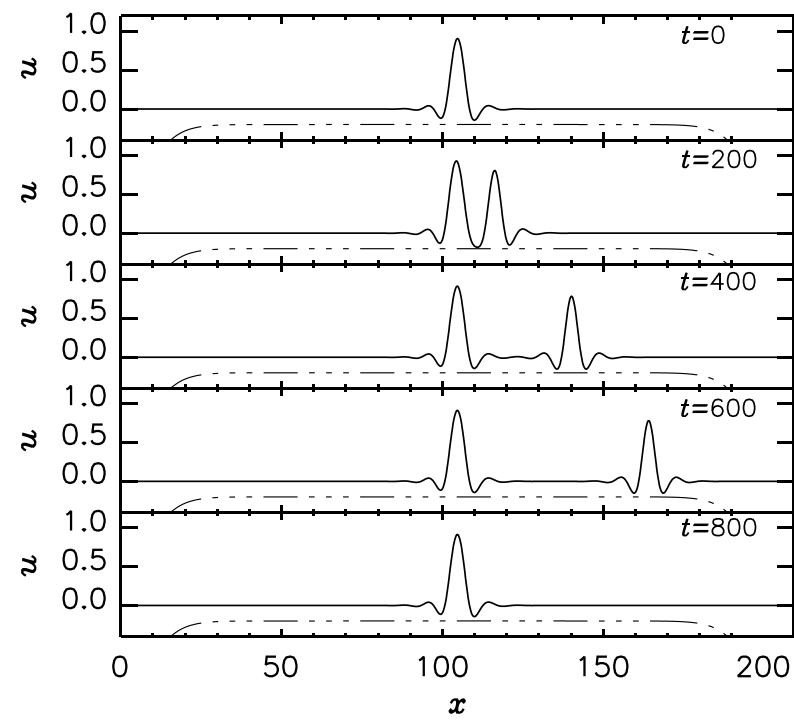

FIG. 11. Type I excitability (SNIC): Region D. An excitable excursion of the DS is shown close to the SNIC for $c=0.12, h=$ 0.085 and $\Delta h=-0.035, \Delta t=10$. Panel (a) shows the contour plot of the real field $u$, while several spatial profiles $u(x)$ for fixed values of $t$ are shown in (b).

supercritical Hopf bifurcation $\mathrm{H}^{-}$(Figs. 5 and 6). Figure 13 shows an example of an excursion in region $\mathrm{C}$, close to $\mathrm{H}^{-}$. Here the resting state is a large-amplitude DS, in contrast to the fundamental solution in region $\mathrm{A}$. Close to $\mathrm{H}^{-}$in region $\mathrm{B}$, DSs exhibit small-amplitude oscillations, but moving further away from $\mathrm{H}^{-}$, a train of DSs is formed through a canard explosion, as explained in the previous section. The excitability threshold is defined by this canard explosion as the transient parameters need to be chosen such that the defect can serve as a source of DSs $(\sim$ train of DSs).

\section{ORGANIZATION OF THE DYNAMICS BY CODIMENSION-2 BIFURCATION POINTS}

In this section, we will show that the various bifurcations involved in the creation of oscillations and excitability, i.e., the Hopf bifurcations $\left(\mathrm{H}^{+}, \mathrm{H}^{-}\right)$and the SNIC, are organized by codimension- 2 bifurcation points. Bifurcation points of codimension-2 require two parameters to be varied for a bifurcation to occur. The various bifurcations unfold from three such codimension- 2 points: two TB points and one SNSL point.

\section{A. Takens-Bogdanov bifurcations}

A Takens-Bogdanov (or double-zero) bifurcation takes place for parameter values $\left(c_{\mathrm{TB}}, h_{\mathrm{TB}}\right)$ if the linearized dynamics
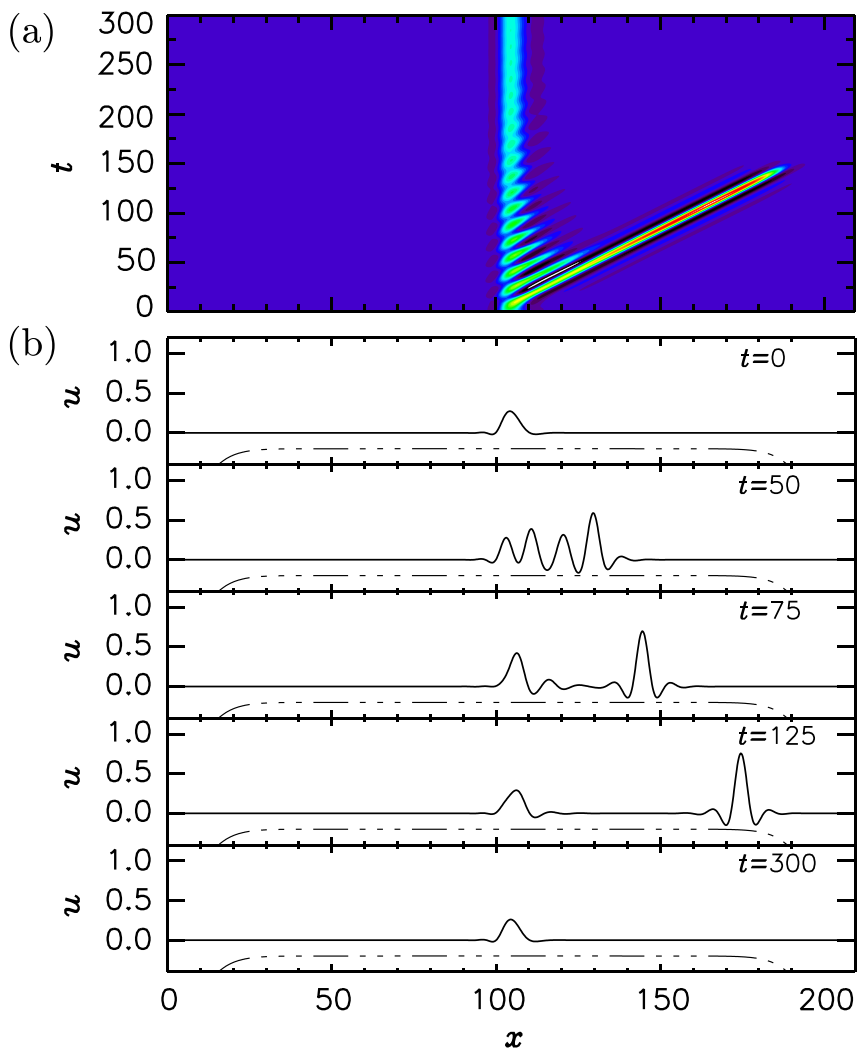

FIG. 12. Type II excitability $\left(\mathrm{H}^{+}\right)$: Region A. An excitable excursion of the DS is shown close to the FC for $c=0.6, h=0.092$ and $\Delta h=0.035 \Delta t=10$. Panel (a) shows the contour plot of the real field $u$, while several spatial profiles $u(x)$ for fixed values of $t$ are shown in (b).

around the fixed point $u_{s, \mathrm{~TB}}$, which is given by the Jacobian matrix, has a zero eigenvalue with algebraic multiplicity 2 $\lambda_{1,2}=0[36,37]$. According to the center manifold theorem, there exists a family of smooth 2-dimensional invariant manifolds $W_{c, h}^{C}$ around the steady state $u_{s, \mathrm{~TB}}$. At that point, $W_{c, h}^{C}$ is tangent to the linear eigenspace spanned by the eigenmodes $\phi_{1}$ and $\phi_{2}$ associated with $\lambda_{1,2}=0$. The projection of our infinite-dimensional dynamical system on the center manifold $W_{c, h}^{C}$ is topologically equivalent to the normal form [29]:

$$
\begin{aligned}
\frac{d A_{1}}{d t} & =A_{2} \\
\frac{d A_{2}}{d t} & =\beta_{1}+\beta_{2} A_{1}+A_{1}^{2}+s A_{1} A_{2},
\end{aligned}
$$

where $A_{1,2}$ represent the amplitudes of the modes $\phi_{1,2}$, and $\beta_{1,2}$ are coefficients which can be determined perturbatively, and the parameter $s$ takes the values \pm 1 . In both cases the TB bifurcation involves a saddle-node bifurcation, Hopf bifurcation, and a SL bifurcation. The SL bifurcation is a global bifurcation and cannot be detected using a local analysis. Therefore, the SL bifurcation has been determined numerically. In a similar notation as we used for Hopf bifurcations, we will denote a $\mathrm{SN}$ bifurcation curve as $\mathrm{SN}^{-}$ if the transverse eigenmode is stable after crossing the center manifold. Likewise, we write $\mathrm{SN}^{+}$when the mode is unstable 

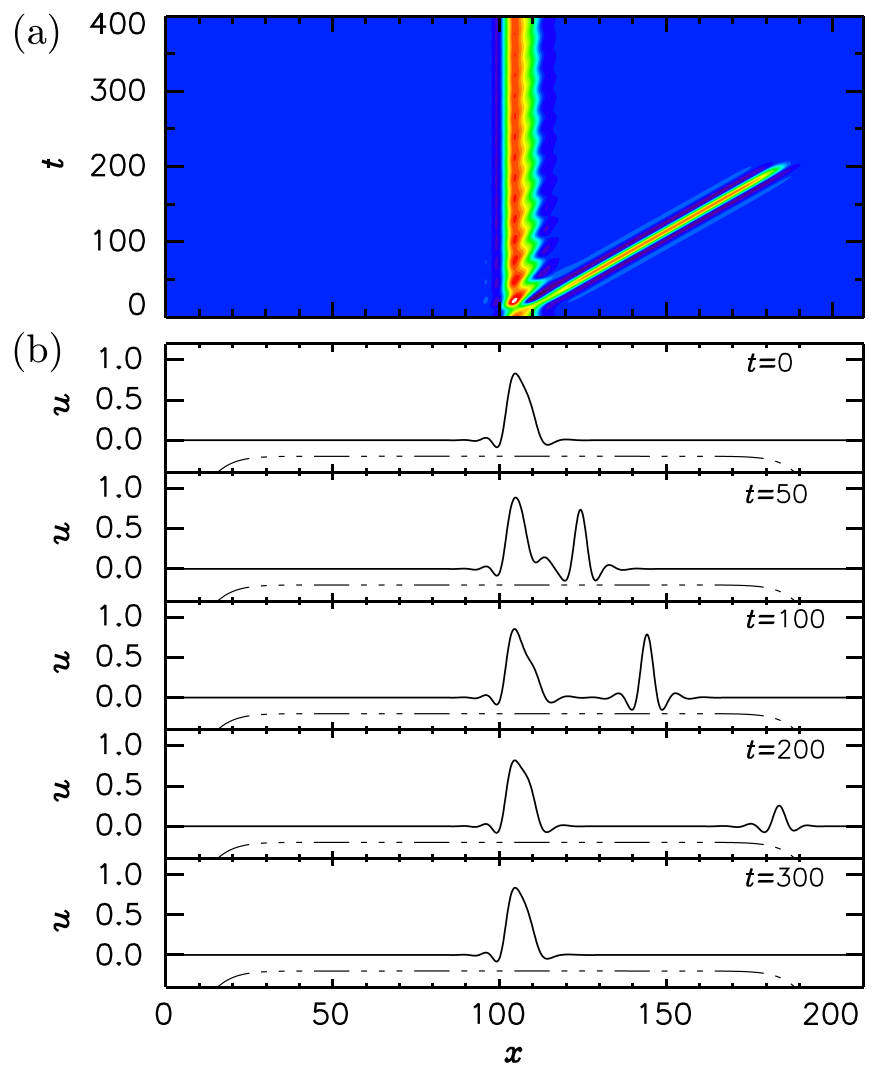

FIG. 13. Type II excitability $\left(\mathrm{H}^{-}\right)$: Region C. An excitable excursion of the DS is shown close to the $\mathrm{H}^{-}$for $c=0.4, h=0.175$ and $\Delta h=-0.045, \Delta t=10$. Panel (a) shows the contour plot of the real field $u$, while several spatial profiles $u(x)$ for fixed values of $t$ are shown in (b).

after crossing the center manifold. For $s=+1$, a subcritical Hopf bifurcation $\mathrm{H}^{+}$unfolds from the TB point, tangent to a saddle-node bifurcation $\mathrm{SN}^{+}$. Furthermore, a $\mathrm{SL}^{+}$, where an unstable cycle is destroyed, is created tangent to the Hopf bifurcation $\mathrm{H}^{+}$. For $s=-1$, a supercritical Hopf bifurcation $\mathrm{H}^{-}$unfolds from the TB point, tangent to a saddle-node bifurcation $\mathrm{SN}^{-}$; and a $\mathrm{SL}^{-}$, where a stable cycle is destroyed, also unfolds tangent to $\mathrm{H}^{-}$.

Figures 14 and 15 show that both types of TB bifurcations occur in the SHE with drift and defect. In Fig. 14 the TB point corresponds to the normal form case with $s=+1$, which we will call $\mathrm{TB}_{1}$. The unfolding of $\mathrm{TB}_{1}$ is further illustrated in Fig. 16, where the top panels show how the bifurcation diagrams change when increasing $c$ and as such crossing $\mathrm{TB}_{1}$. The bottom panels plot the real part of the largest eigenvalues associated with each solution branch. For $c=0.1171$, in Fig. 16(a), the eigenmode transverse to the center manifold is stable, such that we label the saddle-node bifurcation as $\mathrm{SN}_{1}^{-}$. At the $\mathrm{TB}_{1}$ point $(c=0.1173)$, there exist two modes with zero eigenvalue $\operatorname{Re}\left[\lambda_{1,2}\right]=0$ [Fig. 16(b)]. The transverse mode to the center manifold of the $\mathrm{SN}_{1}$ switches from being stable to being unstable, such that we now label the saddle-node $\mathrm{SN}_{1}^{+}$. At the same time, two other bifurcation lines, a subcritical Hopf bifurcation $\left(\mathrm{H}^{+}\right)$and a saddle-loop bifurcation $\mathrm{SL}^{+}$(not shown in Fig. 14) unfold. Figures 16(c) and 16(d) illustrates the situation when further increasing $c$

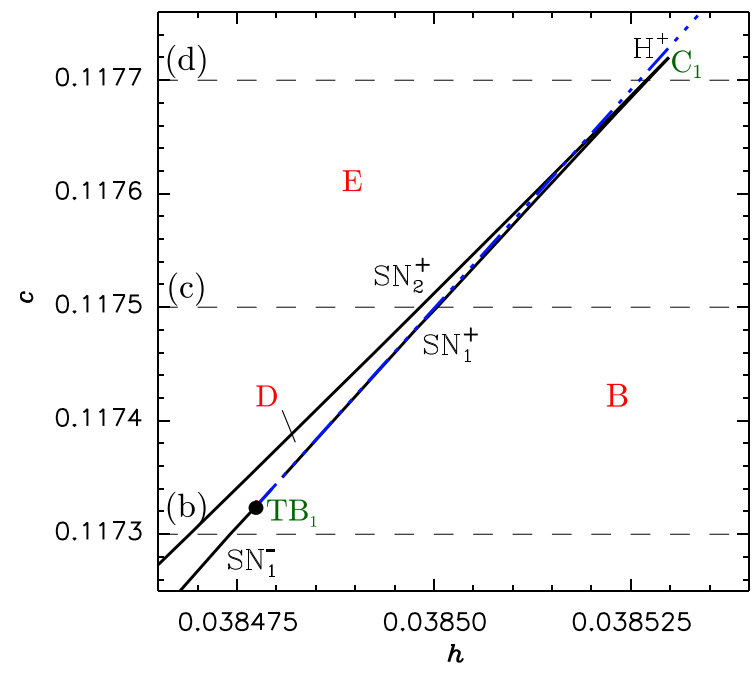

FIG. 14. Unfolding of the Takens-Bodganov $\mathrm{TB}_{1}$ bifurcation, completing the phase diagram in Fig. 5 for $a=1.2$ and $r=-0.2$. The black dashed lines refer to Fig. 16 where the corresponding bifurcation diagrams and eigenvalues are shown. The dot-dashed blue line corresponds to $\mathrm{H}^{+}$.

( $c=0.1175$ and $c=0.1177$ ). The Hopf bifurcation $\mathrm{H}^{+}$moves to the left, destabilizing the branch of fundamental (low bump) solutions [labeled i) in Fig. 2]. $\mathrm{H}^{+}$eventually moves beyond $\mathrm{SN}_{2}^{+}$, and $\mathrm{SN}_{2}^{+}$in its turn approaches $\mathrm{SN}_{1}^{+}$. Increasing $c$ further leads to the coalescence of both unstable $\mathrm{SN}$ curves in a cusp bifurcation $\mathrm{C}_{1}$.

In Figs. 15 and 17, we show the unfolding of the second TB point $\left(\mathrm{TB}_{2}\right)$, corresponding to the normal form case with $s=-1$. Figure 17(a) corresponds to the situation before crossing $\mathrm{TB}_{2}$. Two saddle-node bifurcations can be observed: $\mathrm{SN}_{4}^{-}$leading to a stable structure on the invariant circle and $\mathrm{SN}_{3}^{+}$giving rise to unstable solution. At $\mathrm{TB}_{2}$ (not shown in

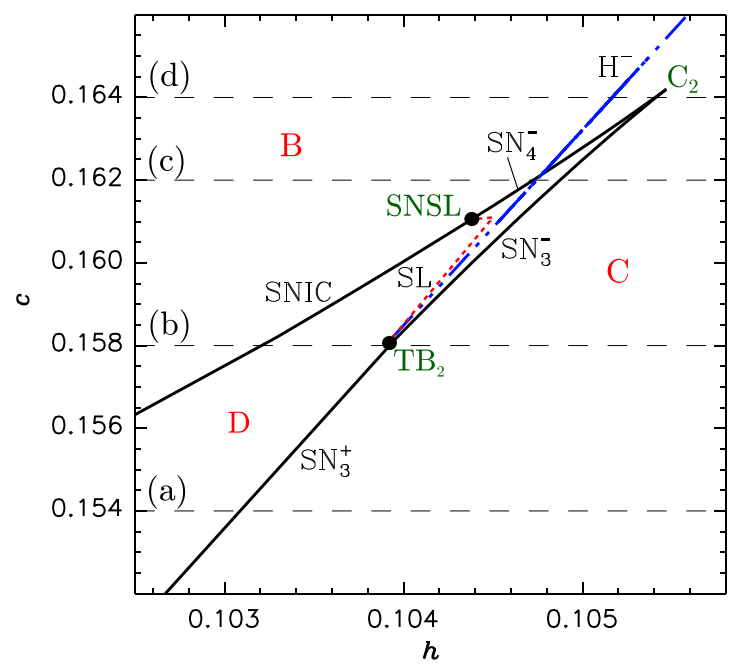

FIG. 15. Unfolding of the Takens-Bodganov $\mathrm{TB}_{2}$ bifurcation, completing the phase diagram in Fig. 5 for $a=1.2$ and $r=-0.2$. The black dashed lines refer to Fig. 17 where the corresponding bifurcation diagrams and eigenvalues are shown. The dot-dashed blue line corresponds to $\mathrm{H}^{-}$and the red dashed line to a SL bifurcation. 

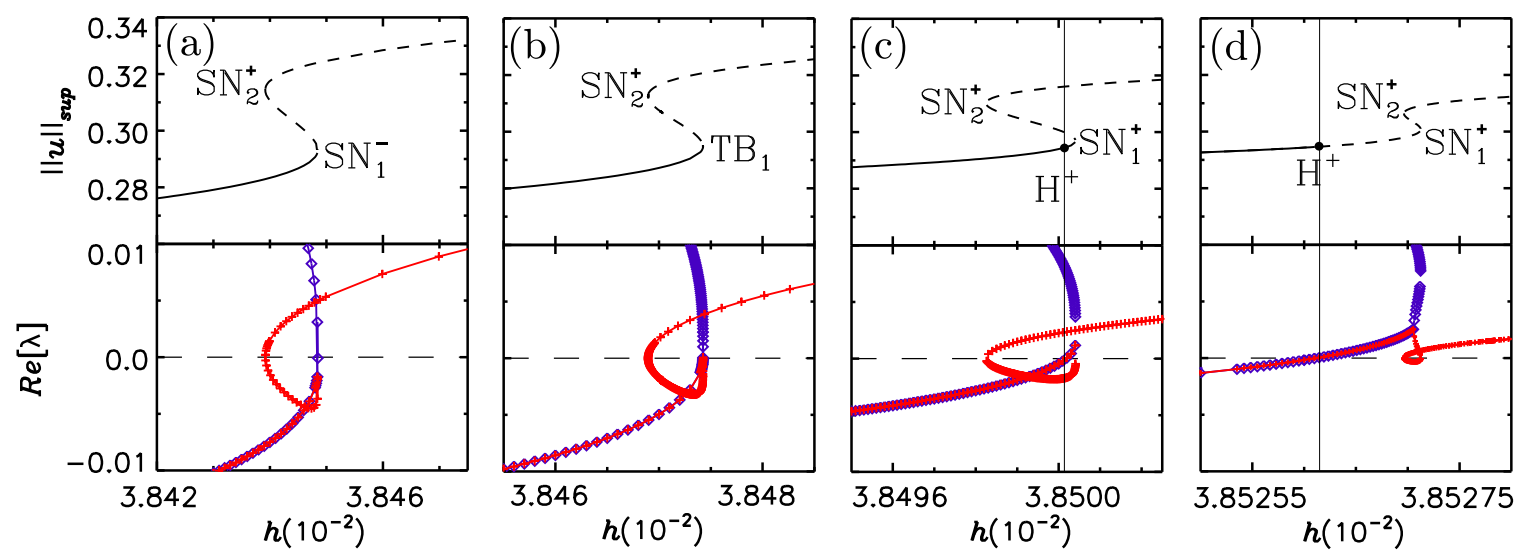

FIG. 16. Bifurcation diagrams as a function of $h$ (top), and the real part of the leading eigenvalues (bottom) for fixed increasing values of $c$ crossing the $\mathrm{TB}_{1}$ point: (a) $c=0.1171$, (b) $c=0.1173$, (c) $c=0.1175$, (d) $c=0.1177$. These values of $c$ are also indicated as horizontal dashed lines in Fig. 14.

Fig. 17), the transverse eigenmode to the center manifold of $\mathrm{SN}_{3}$ crosses the manifold, switching from being unstable to being stable. Therefore, after crossing $\mathrm{TB}_{2}, \mathrm{SN}_{3}^{+}$becomes $\mathrm{SN}_{3}^{-}$. This situation is shown in Fig. 17(b) for $c=0.158$. In this case the Hopf bifurcation curve is supercritical $\left(\mathrm{H}^{-}\right)$and creates a stable cycle which is destroyed in a SL${ }^{-}$bifurcation. As we mentioned before, such SL bifurcations are global bifurcations and cannot be detected locally. We used the scaling law (14) to extrapolate the location in parameter space of $\mathrm{SL}^{-}$, see Fig. 15. Close to the $\mathrm{SL}^{-}$line, SL-mediated Type I excitability can be found in a very narrow region (not shown here). At $c=0.162$, in Fig. $15(\mathrm{c})$, the $\mathrm{SN}_{4}^{-}$already occurs out of the cycle and $\mathrm{H}^{-}$has moved to the left until almost crossing the $\mathrm{SN}_{4}^{-}$. Finally, for $c=0.164, \mathrm{SN}_{3}$ and $\mathrm{SN}_{4}$, both stable, are located very close and they will coalesce in the cusp bifurcation $\mathrm{C}_{2}$.

\section{B. Saddle-node separatrix-loop bifurcation}

The destruction of the SL bifurcation is related with another codimension-2 point known as SNSL [15,38]. A SNSL is a local-global codimension-2 point in which a saddle-node bifurcation takes place simultaneously with a saddle loop bifurcation. Figure 15 shows the unfolding of this SNSL point for the case involving the $\mathrm{SL}^{-}$. One can see that there is a line of saddle-node bifurcations that at one side of the SNSL corresponds to a saddle-node bifurcation out of the limit circle $\left(\mathrm{SN}_{4}\right)$. At the other side, however, it corresponds to a SNIC bifurcation, where the saddle node occurs inside the limit cycle. Similarly as in $\mathrm{TB}_{2}$, the $\mathrm{SL}^{-}$curve also unfolds tangentially to $\mathrm{SN}_{4}$ from the SNSL.

\section{ROBUSTNESS OF THE SCENARIO WITH RESPECT TO PARAMETER VARIATIONS}

In the previous sections, we demonstrated that the interaction between drift and a spatial defect can lead to oscillatory and excitable dynamics of a DS. We explored these phenomena by focusing on the SHE using one parameter set (i.e., $a=1.2$ and $r_{0}=-0.2$ ). For this set of parameters we analyzed all bifurcations, both those of codimension- 1 and the codimension-2 Takens-Bogdanov points serving as organizing centers of the dynamics. In this section, we show that these dynamical regimes are a general feature when including drift and defect in a broad parameter range. In order to check the consistency of the scenario in the SHE, we studied the
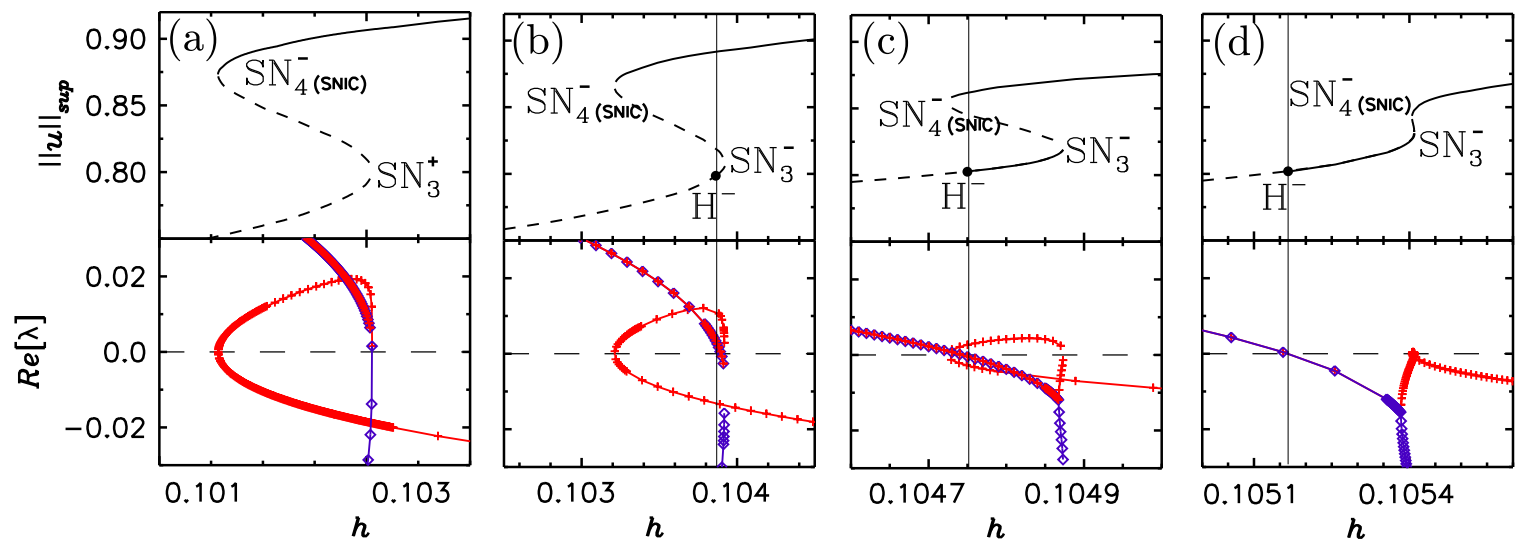

FIG. 17. Bifurcation diagrams as a function of $h$ (top), and the real part of the leading eigenvalues (bottom) for fixed increasing values of $c$ crossing the $\mathrm{TB}_{2}$ point: (a) $c=0.154$, (b) $c=0.158$, (c) $c=0.162$, and (d) $c=0.164$. These values of $c$ are also indicated as horizontal dashed lines in Fig. 15. 


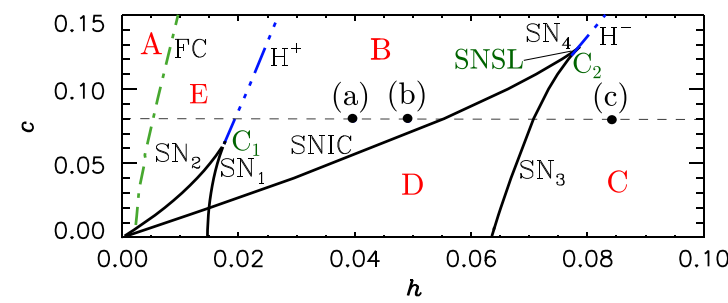

FIG. 18. Two-parameter ( $c$ vs $h$ ) phase diagram of the system for $a=1$ and $r=-0.09$. The bifurcation curves and the labels of the regions corresponds to the ones in Fig. 5. The points labeled with (a), (b), and (c) correspond to the solutions shown in Fig. 19 for $c=0.08$.

location of the previously characterized bifurcations for an alternative parameter set: $a=1$ and $r=-0.09$. In Fig. 18 we plot the phase diagram corresponding to those values. The same bifurcation curves and dynamical regions are found as in Fig. 5. Therefore, it is not too surprising that the dynamics is largely similar as previously reported.

Figure 19 shows the temporal evolution of several trains of solitons corresponding to different values of $h$ for $c=0.08$. Figure 19(a) shows a train of solitons for $h=0.04$ found right in the middle of oscillatory region B in Fig. 18. Similarly as in Fig. 9, the period of oscillations of the DS pinned at the defect increases as one approaches the SNIC bifurcation, and a fast and slow time scale can be observed [Fig. 19(b)]. Moreover, an additional DS is regularly emitted slightly to the right of the defect. There are thus two competing oscillations: One is related with the emission of a DS at the spatial defect (and its period diverges when approaching the SNIC), and the other is related with the emission of a DS at the first oscillation of the tail of the pinned DS. This second oscillation has a period
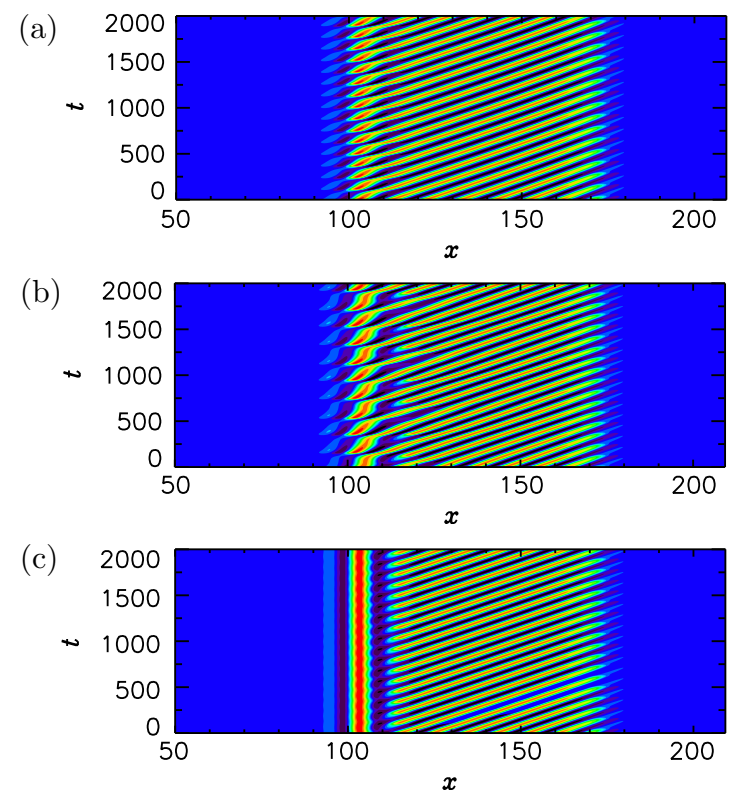

FIG. 19. Contour plots of $u(x, t)$ showing the spatiotemporal evolution of oscillatory solutions for $c=0.08$ (see dashed line in Fig. 18). The defect strength is varied: $h=0.04$ (a), 0.05 (b), 0.085 (c). which increases when decreasing $h$ within region B, potentially because there exists a secondary SNIC or SL bifurcation.

So far, the dynamics are similar to those reported in Figs. 9 and 5 for $a=1.2$ and $r=-0.2$. However, when increasing $h$ beyond the SNIC bifurcation, some differences can be observed. For $a=1.2$ and $r=-0.2$, the only attractor of the system in region $\mathrm{C}$ was a single peak DS centered at the spatial defect (Fig. 5). This solution exists in region $\mathrm{C}$ for $a=1$ and $r=-0.09$ as well (Fig. 17), but here the system shows bistability between this single pinned DS and the oscillatory solution shown in Fig. 19(c). This new limit cycle is composed of a small amplitude oscillation of the DS centered at the spatial defect and a train of solitons emitted from the first spatial oscillation of its tail [this oscillation was already present in Fig. 19(b)]. This added layer of complexity could be due to the fact that for these parameters, the pinning range of DSs and the Turing instability of the background $u=0$ state are very close in parameter space. Therefore, DSs can be triggered not only at the spatial defect but also from the tails of the DS.

\section{SPATIAL DEFECT IN THE GAIN TERM}

In this section we consider the SHE with drift and defect, where the drift is introduced in the same way as before in Eq. (10), but the spatial defect is now added to the linear gain term. The resulting SHE is given by:

$$
\partial_{t} u=[r(x)+b(x)] u+a u^{2}-g u^{3}-\left(\partial_{x}^{2}+k_{0}^{2}\right)^{2} u-c \partial_{x} u,
$$

where the spatial inhomogeneity is no longer an independent driving term in the equation [as in Eq. (10)], but it is part of the gain parameter that now reads $\tilde{r}(x)=r(x)+b(x)$. In other words, the effective gain parameter $\tilde{r}$ changes around the center $x_{0}$ due to the effect of the defect and close to the borders due to the absorbing boundary conditions. We will show that in this situation the bifurcation scenario is richer and more involved. However, despite the added complexity, the competition between drift and defect still introduces similar oscillatory and excitable dynamics as before, confirming the generality of the concepts presented here. As in Sec. III D, we will consider $g=1, a=1.2$, and $k_{0}^{2}=0.5$.

In the present case, both DSs consisting of a single peak and multiple peaks are involved in the bifurcation diagram, in particular two-peak states and asymmetric rung states [39]. The rung states connect the single peak with the two-peak DSs. The existence of a pinning defect implies that these solutions can be pinned at different locations of the DS profile. For $c=0$, DSs pinned outside the center of its profile are degenerate with a branch of solutions corresponding to DS pinned at the left of the center and another branch corresponding to DS pinned at the right. Figure 20 represents the bifurcation diagram for a value $c=0.0001$. In order to make the diagram easier to understand, we plot the two-peak DSs branches in blue, the single DSs branches in black, and the asymmetric rung states branches in red. For $c=0$, six transcritical bifurcations $\left(\mathrm{T}_{1}, \ldots, \mathrm{T}_{6}\right)$ take place at $h=0$ where the solution branches involved exchange their stability. Considering a finite value for $c$ (as in Fig. 20) leads to three effects. First, the transcritical bifurcations at $h=0$ become imperfect and branches at positive and negative values of $h$ detach in a similar way 


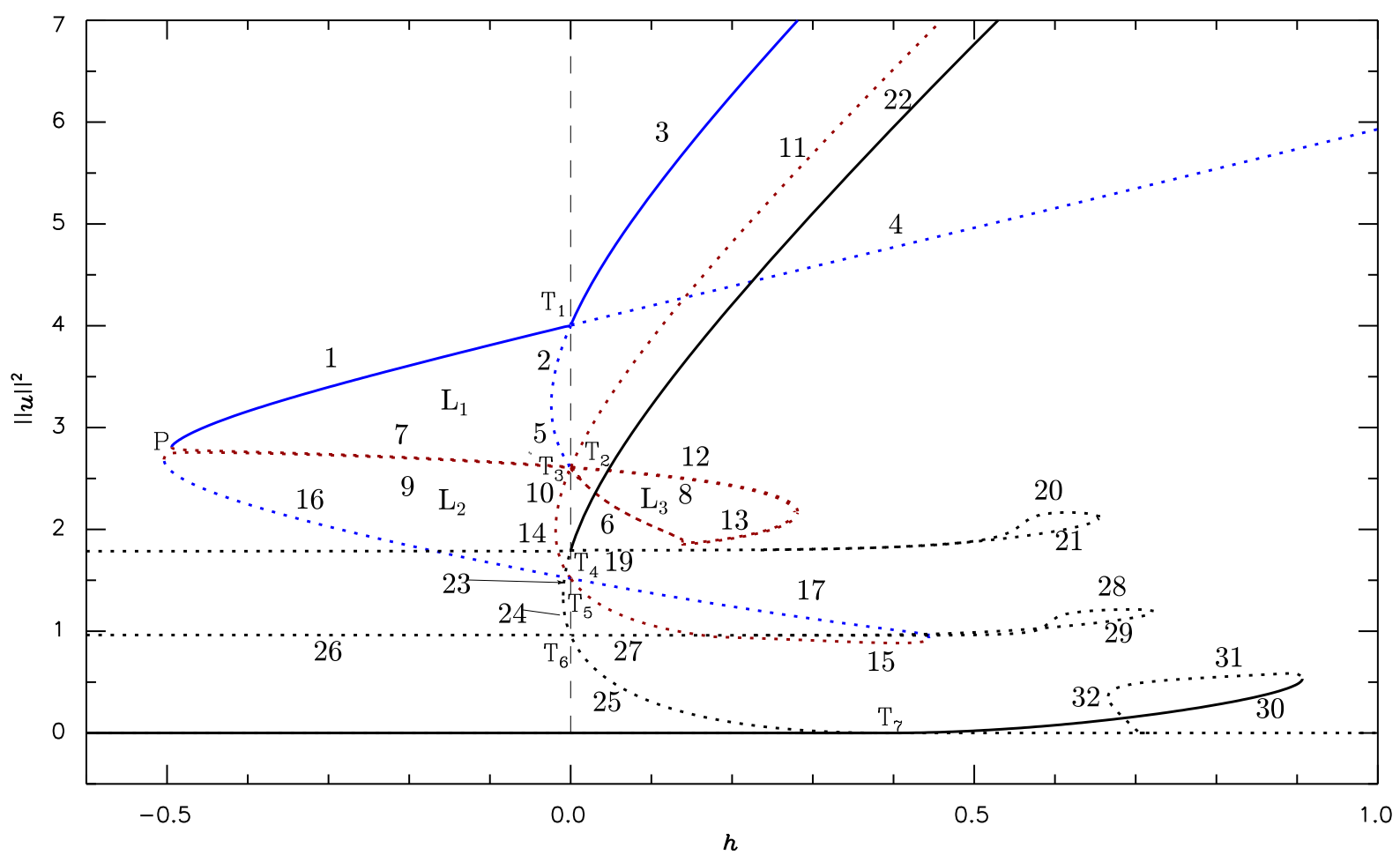

FIG. 20. Bifurcation diagram for Eq. (18) with the defect in the gain term at $c=0.0001$. Branches corresponding to DSs with two peaks are shown in blue, DS with a single peak in black, and the asymmetric rung states in red. Solid and dashed lines represent stable and unstable states, respectively.

as in Sec. III D. Second, the branches of solutions pinned at the left or at the right of the center are no longer degenerate. Third, the pitchfork bifurcation $\mathrm{P}$ at negative values of $h$ where rung states are born from two peaks DS becomes imperfect [for a more detailed picture see Fig. 28(a) in the Appendix]. Furthermore, the imperfect bifurcations arising at finite $c$ lead to the reconnection of different branches forming isolas (loops) as described in the Appendix.

Now the fundamental solution of Eq. (18) is the trivial one $u=0$ without any deformation. This fundamental solution undergoes a transcritical bifurcation at $h>0$, labeled $\mathrm{T}_{7}$ in Fig. 20, where it exchanges stability with branches 25 and 30 corresponding to small bump states with one and two peaks, respectively. The other main attractors are those corresponding to the branches 1, 3, 22, and 30 and can be seen in Fig. 21. They consist in a two-peak DS pinned at its center minimum, a two-peak DS pinned at the right peak (and another one pinned on the left peak), a single DS pinned at its center, and a small amplitude two-peak DS pinned at its central minimum.

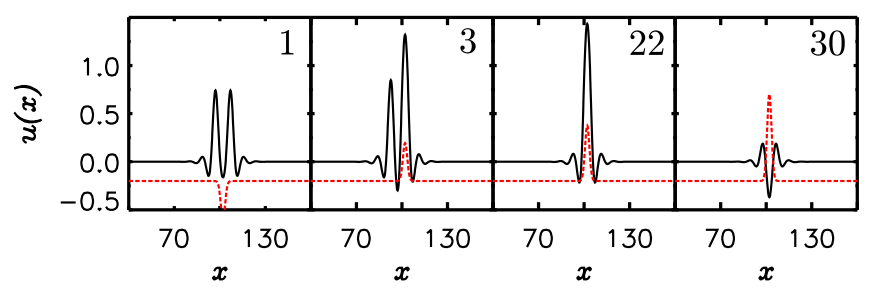

FIG. 21. Spatial profiles $u(x)$ of the main attractors of the system for $c=0$. The red dashed line shows the defect profile.
A complete analysis of the states and reconnection of branches related with this scenario is presented in the Appendix. Here, for simplicity, we skip the unnecessary details and we focus on the study of branches 11, 12, 13 and 22, related with the one-peak DS, which are the ones necessary to explain the oscillatory and excitable dynamics in the system.

For very small values of the drift $c$, branches 11,12 and 13 are disconnected from branch 22, as shown in Fig. 20. However, as $c$ increases, branch 19 , which is connected to branch 22, approaches branch 13 and reconnects with it for $c=0.0003$ (see Appendix). As a consequence for $c>0.0003$ branch 22 is connected to branch 11 via branches 13 and 12 as shown in Fig. 22(a) for $c=0.05$. The spatial profiles of the DSs corresponding to these branches are shown in Fig. 22(d).

As the drift strength $c$ is further increased branch 11 moves to the left of branch 12 and $\mathrm{SN}_{1}$ becomes a SNIC bifurcation [Fig. 22(b)]. Similarly as in Eq. (10), this SNIC bifurcation leads to oscillations of the high amplitude DS (region B). An example of these oscillations, corresponding to a train of solitons, is shown in Fig. 23. Close to the SNIC the period of the oscillations is very large and one observes the typical timescale separation. The period decreases for decreasing values of $h$ moving away from the SNIC. Decreasing $h$ even further, the oscillations disappear in a fold of cycles (FC). The stable limit cycle (train of solitons) collides with an unstable limit cycle created at the subcritical Hopf $\mathrm{H}^{+}$of the trivial solution. Increasing $c$ further $(c=0.3), \mathrm{SN}_{2}$ and $\mathrm{SN}_{3}$ coalesce in a cusp bifurcation $\mathrm{C}$, while the SNIC bifurcation remains present up to higher values of $c$ shown in Fig. 22(c).

In the case of Eq. (10), we demonstrated in Sec. V that both Type I and Type II excitability originated close 

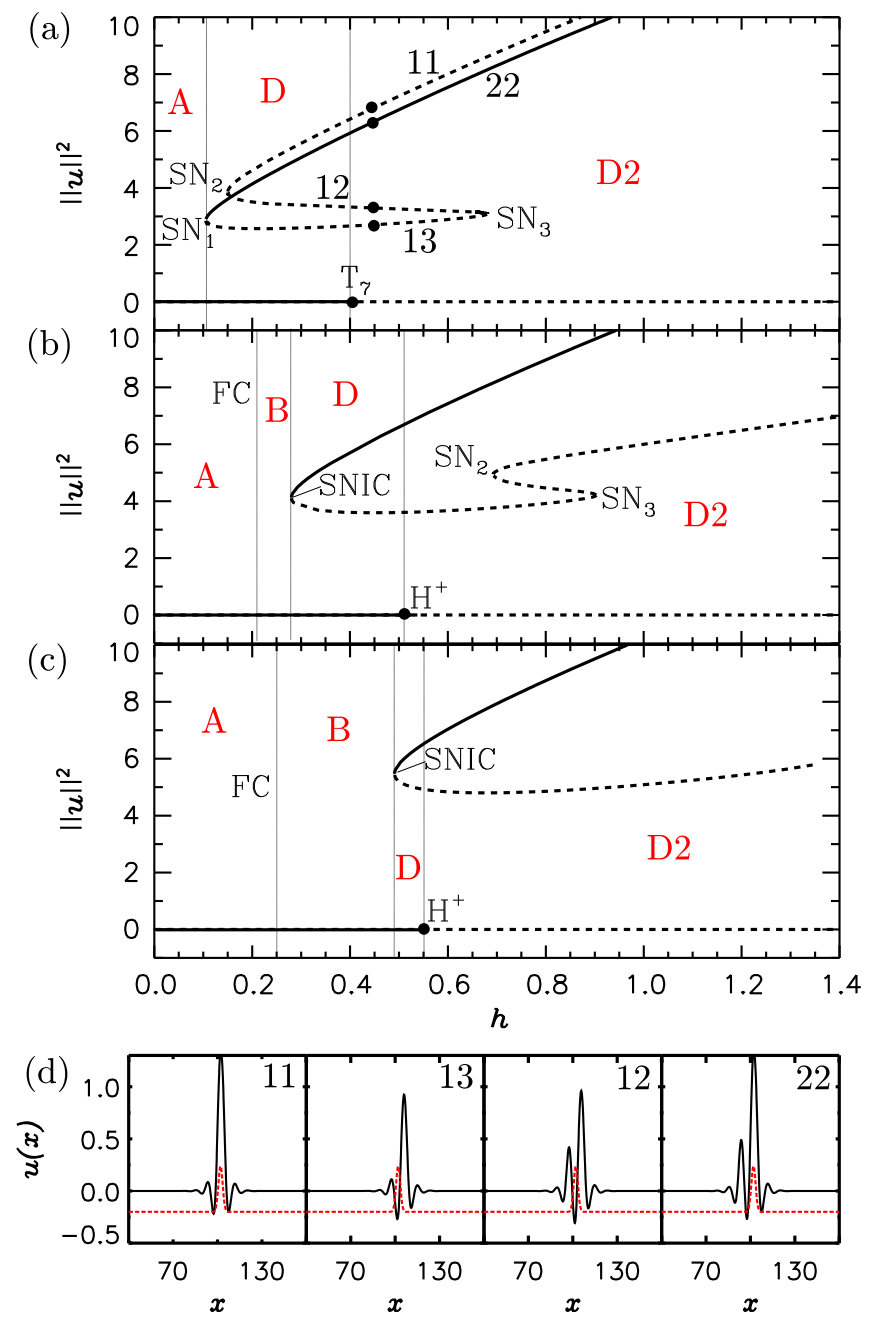

FIG. 22. Bifurcation diagrams for Eq. (18) with the defect in the gain term for increasing values of the drift $c: c=0.05$ (a), $c=$ 0.15 (b), and $c=0.3$ (c). Only the branches for $h>0$ relevant to the discussion are shown. The main dynamical regimes are indicated in red and explained in the main text. Panel (d) shows the spatial profiles of the main attractors for $c \neq 0$.

to the SNIC bifurcation, Hopf bifurcations, and the fold of cycles. Similar bifurcations occur here when the spatial defect is present in the gain term, such that one can expect

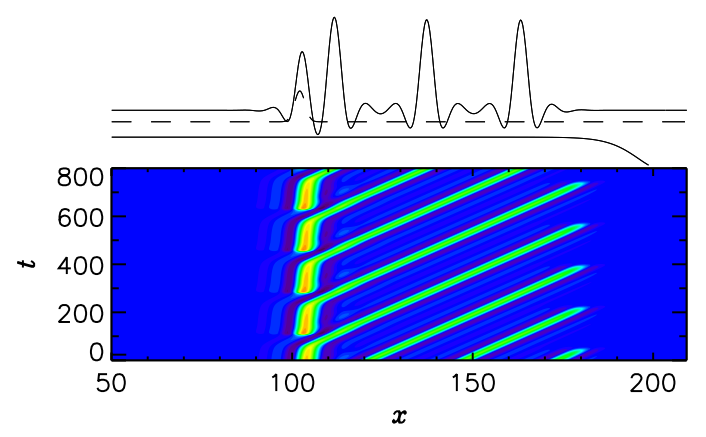

FIG. 23. Contour plots of $u(x, t)$ showing the spatiotemporal evolution of oscillatory solutions of the high amplitude DS (train of solitons) for Eq. (18) with the defect in the gain term for $c=0.15$ and $h=0.268$. Above the contour plots the spatial profile $u(x, t=800)$ is plotted. (a)

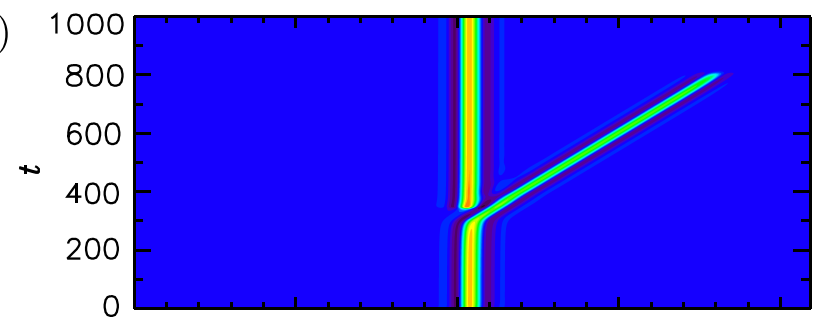

(b)

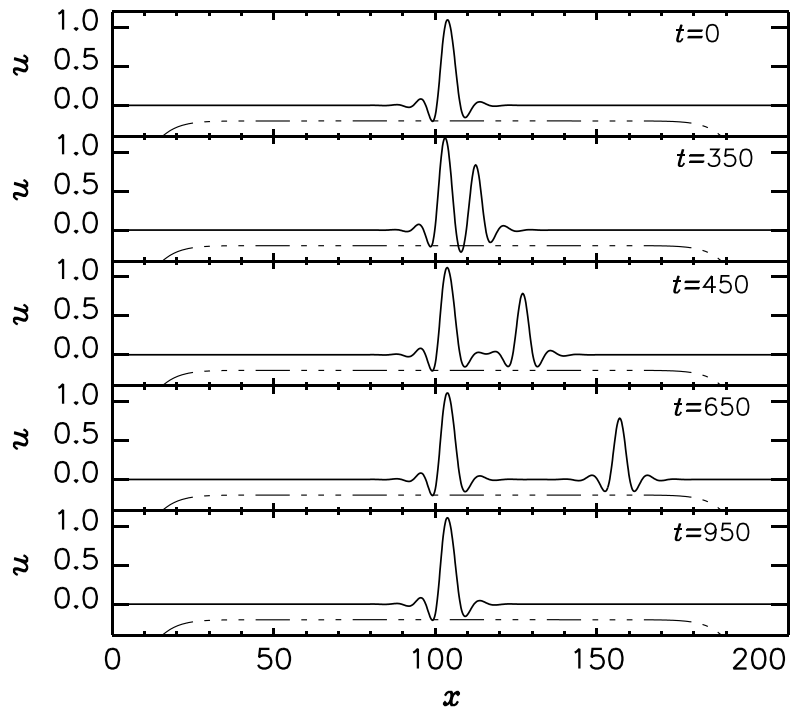

FIG. 24. Excitable excursion of a DS for Eq. (18) with the defect in the gain term. The parameters are $c=0.15, h=0.281, \Delta h=$ -0.03 , and $\Delta t=10$. Panel (a) shows the contour plot of $u(x, t)$ illustrating the spatiotemporal evolution of the excited DS. In (b) several snapshots of spatial profiles are shown for fixed values of $t$.

to again find excitability of the high-amplitude DS solution. Figure 24 shows that this is indeed the case. Close to the SNIC bifurcation, Type I excitability is observed by transiently perturbing the parameter set. This way, the system transiently finds itself in the oscillatory region and at the defect location a new DS is emitted and pulled towards the boundary, where it is removed from the domain.

Finally, Fig. 25 shows the phase diagram with the organization of the various bifurcations in the parameter space ( $h$ and $c$ ). The black lines show instabilities associated to the large-amplitude DS while blue lines show instabilities of

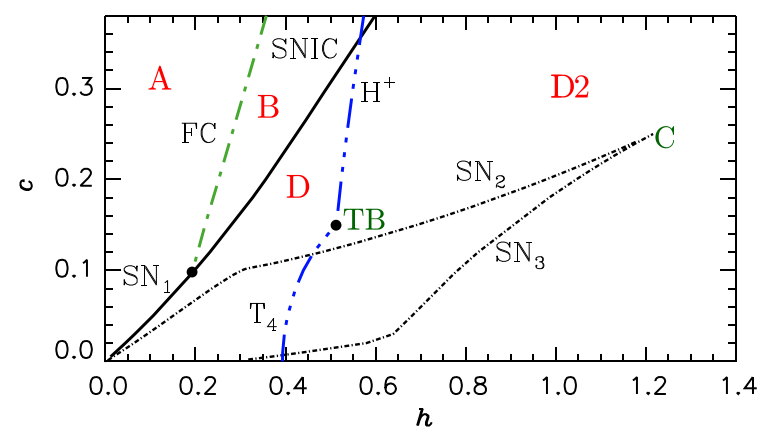

FIG. 25. Two-parameter ( $c$ vs $h$ ) phase diagram for Eq. (18) with the defect in the gain term. The bifurcations and regions A-D are explained in the main text. 
the trivial solution $u=0$. The subcritical Hopf bifurcation $\mathrm{H}^{+}$is shown to again unfold from a TB point. Therefore, the trivial solution that became unstable at $\mathrm{T}$ for values of $c$ below TB will become unstable at $\mathrm{H}^{+}$for values of $c$ above TB. Four dynamical regions, labeled $\mathrm{A}$ to $\mathrm{D}$ are denoted in the phase diagram. These regions allow us to identify where stable solutions can be found and where they show oscillatory or excitable dynamics:

Region A: The trivial solution is stable. This region is similar to Region A in Fig. 5. For parameters close to the FC line the system can display Type II excitability.

Region B: The system displays DSs which oscillate periodically in time, similarly as in Region B of Fig. 5.

Region D: The system admits stable large amplitude pinned DSs coexisting with the trivial solution $u=0$. Within this region, the system can display Type I excitability.

Region D2: This region is similar to Region D, but the trivial solution is now unstable. For $c>0.35$, region D2 borders region $\mathrm{B}$. As a consequence, for parameter values within region D2 close to the SNIC line, the system can display Type I excitability.

\section{DISCUSSION}

In this work, using the Swift-Hohenberg equation for a real field, we presented a mechanism to induce dynamical instabilities of otherwise static DSs, preserving the structure of the DS. The mechanism relies on the interplay between spatial inhomogeneities and drift, together with absorbing boundary conditions, and therefore can be implemented under very general conditions. The presence of a defect and drift introduces two competing effects. On the one hand, a defect pins a DS at a fixed position, while, on the other hand, the drift tries to pull it out. If the drift overcomes the pinning force, DSs are released from the inhomogeneity. Depending on the strength of the spatial defect and the strength of the drift, we found three main dynamical regimes: (i) stationary (pinned) DS solutions; (ii) oscillatory regimes, where the pinned DS serves as continuous source of drifting DSs; and (iii) excitability, where a perturbation may trigger a single DS that drifts away from the defect location. The excitability regime requires the presence of absorbing boundary conditions, which removes the drifting DS. For systems with periodic boundary conditions the drifting DS are reinjected and a train of solitons is typically observed instead [40]. We first reported on these results in Ref. [17]. Here we presented a detailed bifurcation analysis, we linked these bifurcations to the presence of oscillatory and Type I and Type II excitable dynamics of DSs, and we analyzed how these dynamics are intimately linked to the presence of various codimension-2 points such as Takens-Bogdanov bifurcations and saddle-node separatrix loops. Next, we set out to show that this mechanism generating oscillations and excitability is generic, such that our analysis sheds light on the influence of defects and drift in any physical system. We addressed this question of generality in two ways: (i) we showed that the dynamics persisted for different parameter sets in the SHE, (ii) we changed the SHE by introducing the defect in the gain term rather than including it as an independent driving term, and we demonstrated that oscillations and excitability were generated in a similar way. Furthermore, we have also shown recently that in different nonvariational equations, such as the Lugiato-Lefever equation for a nonlinear Kerr optical cavity, the interaction of drift and defect can generate similar oscillatory dynamics [40]. Therefore the scenario described here leading to spatiotemporal dynamics of DSs does not depend on the details of the system.

We believe that our work provides a solid theoretical framework to explain the dynamics of DSs in systems with drift and defect. Our analysis could be especially useful for the field of optics. In optics, DSs appear in nonlinear optical resonator as structures that are exponentially localized in space and/or time, and they are known as cavity solitons (CSs). Spatial CSs can appear in ring cavities or Fabry-Perot interferometers filled with a nonlinear medium when they are pumped by an external source [7]. Temporal CSs appearing in fiber cavities or microresonators have recently attracted a lot of interest due to the strong correspondence between Kerr temporal solitons and frequency combs (FCs) [41]. FCs consist in a set of equidistant spectral lines that can be use to measure light frequencies an time intervals more easily and precisely than ever before $[42,43]$. For this reason FCs open a large variety of applications going from optical clocks to astrophysics [43]. Furthermore, CSs have long been proposed as bits for all-optical memories [7,44-47], due to their spatial localization and bistable coexistence with the fundamental solution.

In optical systems the drift can be produced by misalignments of mirrors [20,21], nonlinear crystal birefringence [22], parameter gradients [23], or by higher-order effects chromatic light dispersion [48]. Inhomogeneities can originate from mirror or waveguide imperfections in an optical cavity and from the presence of fiber impurities, leading to variations in absorption coefficient or refractive index [49-51]. Synchronously pumped fiber cavities have also been shown to be modeled by a Lugiato-Lefever equation with a well-defined inhomogeneity in the pump [52,53]. The drift-defect mechanism can explain experimental observations in semiconductor microresonators $[45,54]$ and could be applicable to a wide variety of other optical systems. We finally remark that the mechanism for excitability reported here is generic and therefore it can take place in a variety of systems beyond optics provided that there are spatial inhomogeneities and drift.

\section{ACKNOWLEDGMENTS}

This research was supported by the Research FoundationFlanders (FWO-Vlaanderen), by the Junior Mobility Programme (JuMo) at KU Leuven, by the Belgian Science Policy Office (BelSPO) under Grant No. IAP 7-35, by the Research Council of the Vrije Universiteit Brussel, by the Ministerio de Economía y Competitividad (Spain) and Fondo Europeo de Desarrollo Regional under Grants Intense @ Cosyp (FIS2012-30634) and TripHop (TEC2012-36335).

\section{APPENDIX}

In this Appendix we analyze in detail the scenario in Sec. VIII, in particular the transition that takes place when drift is considered. As we said in the main text, at $h=0$ the 


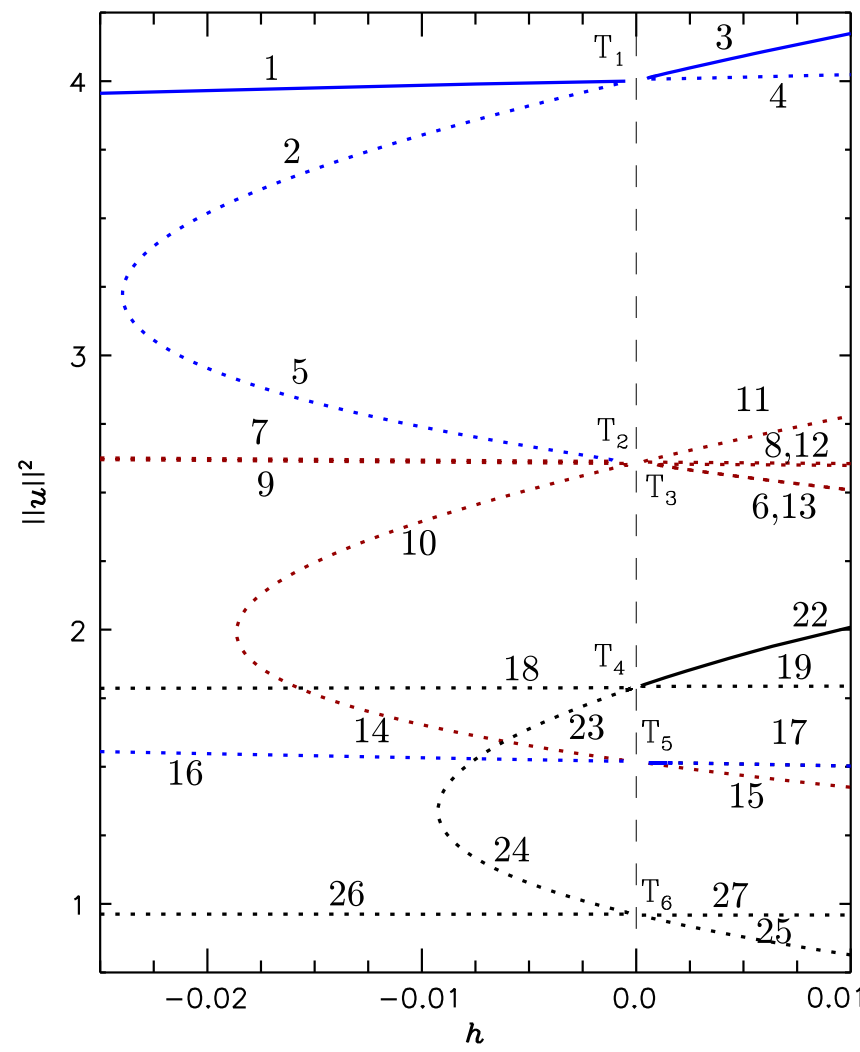

FIG. 26. Detail of the diagram in Fig. 20 showing the imperfect transcritical bifurcations around $h=0$. Branches corresponding to DSs with two peaks are shown in blue, DS with a single peak in black, and the asymmetric rung states in red. Solid and dashed lines represent stable and unstable states, respectively. $\mathrm{T}_{1}, \ldots, \mathrm{T}_{6}$ represent transcritical bifurcations.

transcritical bifurcations become imperfect and branches for $h>0$ and $h<0$ detach. In Fig. 26 a zoom of the diagram displayed in Fig. 20 for $c=0.0001$ shows the imperfect transcritical bifurcations around $h=0$. The imperfect transcritical bifurcation labeled as $\mathrm{T}_{1}$ involves only two-peak states; $\mathrm{T}_{4}$ and $\mathrm{T}_{6}$ involve single-peak DS; and $\mathrm{T}_{2}, \mathrm{~T}_{3}$, and $\mathrm{T}_{4}$ a combination of rung states and two-peak states. In the following we show in detail the unfolding of the transcritical bifurcations $\mathrm{T}_{2}, \mathrm{~T}_{3}$, and $\mathrm{T}_{4}$ due to the drift. Branches 5, 6, 7, and 8 are those related with $\mathrm{T}_{2}$, and $9,10,11$, and 12 are related with $\mathrm{T}_{3}$. Analyzing these bifurcations in more detail (see Fig. 27), we find that there is a doublet of transcritical bifurcations, one related with the rung states pinned at the right (labeled $\mathrm{T}_{2,3}^{R}$ ), and one related to the solutions pinned on the left (labeled $\mathrm{T}_{2,3}^{L}$ ), which for $c=0$ are degenerate in norm. When the drift is included, the diagram in Fig. 27 shows that those bifurcations become distinguishable and therefore, we can differentiate between rung states pinned on the left, L states (plotted with pointed lines) and rung states pinned on the right $\mathrm{R}$ states (plotted in dashed lines). The bottom panel in Fig. 27 shows the profiles corresponding to the L states. In black dashed lines we show how the branches are connected through $\mathrm{T}_{2}$ and $\mathrm{T}_{3}$ for $c=0$.

The diagram shown in Fig. 28 represents the transcritical bifurcation $\mathrm{T}_{4}$ and its imperfection corresponding to the diagram in Fig. 26. In this bifurcation only branches 18, 19,
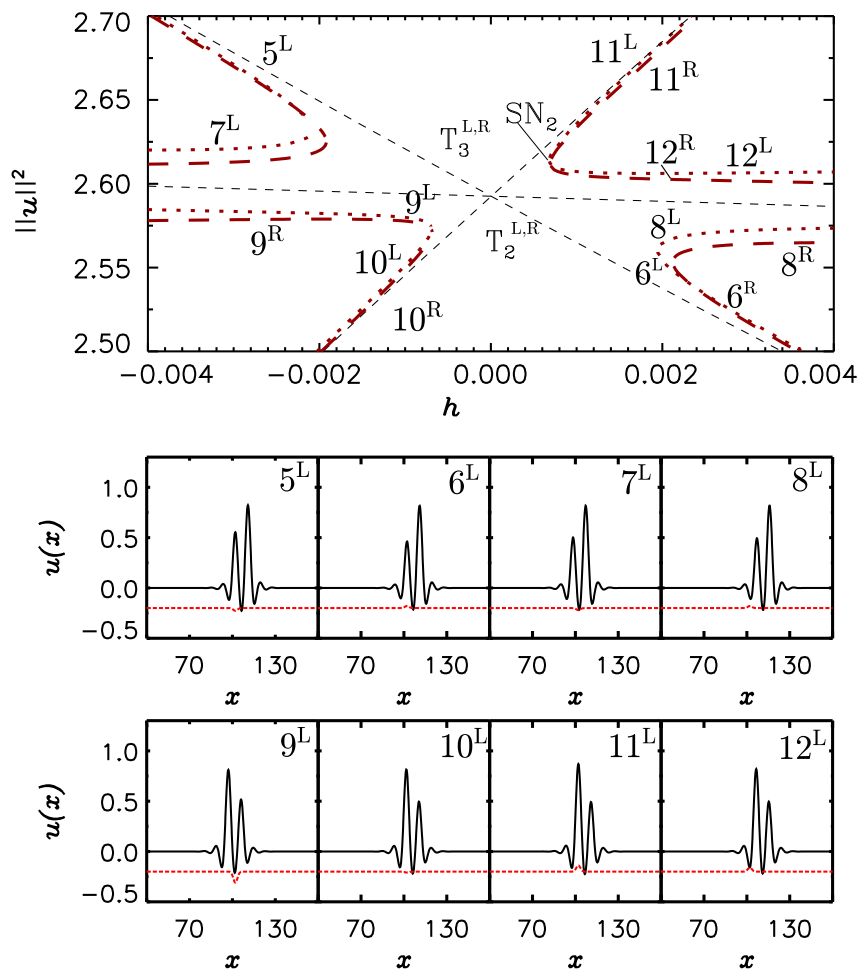

FIG. 27. Transcritical bifurcations $\mathrm{T}_{2}$ and $\mathrm{T}_{3}$ and their imperfections corresponding to Fig. 26 are shown, together with examples of the spatial profiles of the $\mathrm{L}$ states. The two red (dotted and dashed) lines correspond to the bifurcation for $c \neq 0$, while the black dashed lines correspond to the overlapping solution branches for $c=0$.

22 , and 23, corresponding to the single-peak solitons shown in the bottom panel, are involved. In this case we only show the branches corresponding to the L states, although, as before, $\mathrm{R}$
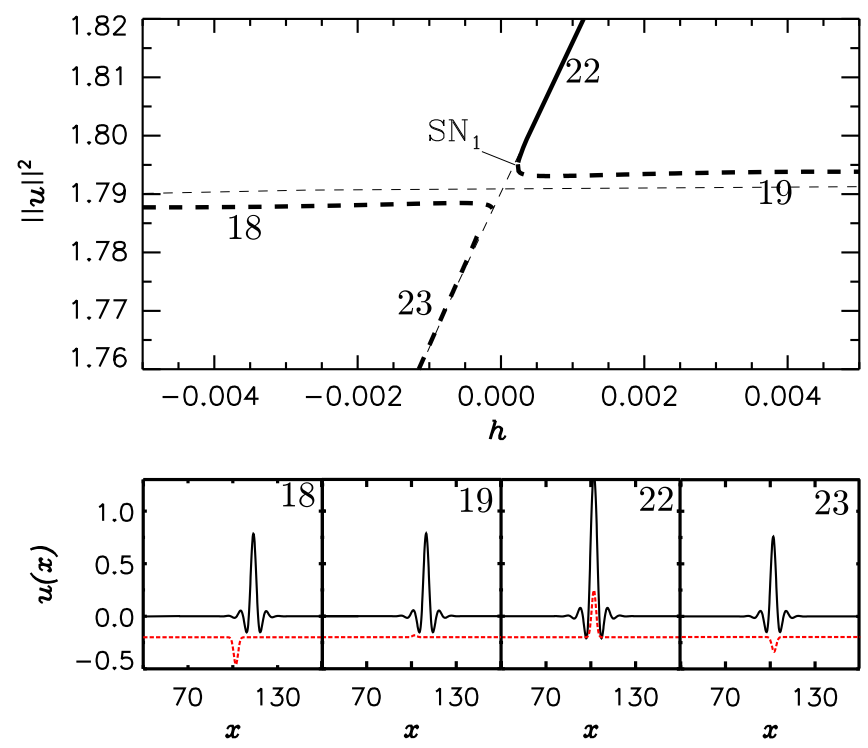

FIG. 28. Bifurcation diagram showing the transcritical bifurcation $\mathrm{T}_{4}$ and its imperfection for $c=0.0001$. In the bottom panel the profiles of the states corresponding to those branches are shown. The thin dashed lines represent the solution branches at $c=0$. 


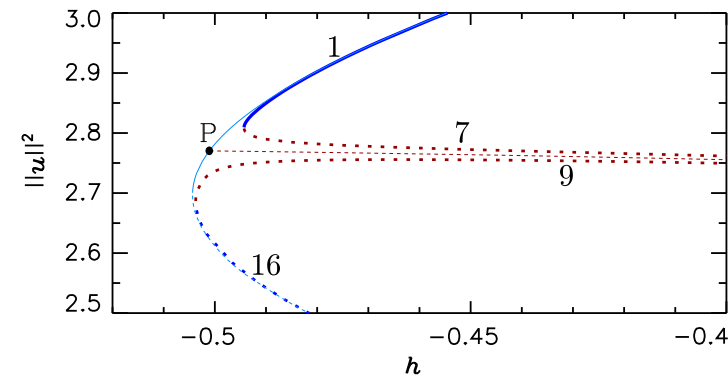

FIG. 29. Pitchfork bifurcation (P) for $c=0$ and its imperfection for $c=0.0001$ are shown.

states would be also present. As a result of the imperfection of $\mathrm{T}_{4}$ and $\mathrm{SN}_{1}$ are created.

It is known that the rung states are asymmetric solutions connecting the solution branches with an even number of peaks with those with an odd number of peaks in the homoclinic snaking [39]. The connection of those rung states branches with the snaking occurs through a Pitchfork bifurcation. Due to the breaking of $x$ reversibility (for instance, by including a drift term in the system) that pitchfork bifurcation becomes imperfect and results in the formation of isolas [35]. In our case, we are locally modifying the strength of the gain parameter $r$, and therefore there is the possibility of reaching the previous pitchfork bifurcation. This is what happens for $h<0$, as shown in Fig. 20. In the diagram of Fig. 29 the pitchfork bifurcation $\mathrm{P}$ is plotted in more detail and becomes imperfect when $c \neq 0$. For $c=0$, branches 7 and 9 corresponding to the rung states are degenerate in norm, but when $\mathrm{P}$ becomes imperfect the degeneration disappears and the solution branches become distinguishable. This imperfect pitchfork and the imperfect transcritical bifurcations $\mathrm{T}_{1}, \mathrm{~T}_{2}, \mathrm{~T}_{3}$, and $\mathrm{T}_{5}$ are responsible for the formation of isolas $\mathrm{L}_{1}$ (composed by branches $1,2,5$, and 7) and $\mathrm{L}_{2}$ (composed by 9, 10, 14, and 16) in Fig. 20. When the strength of the drift further increases, the isolas shrink until eventually they disappear $[35,48]$.

Finally, in order to understand the transition between the diagrams in Figs. 20 and 22(a), we need to see how branches

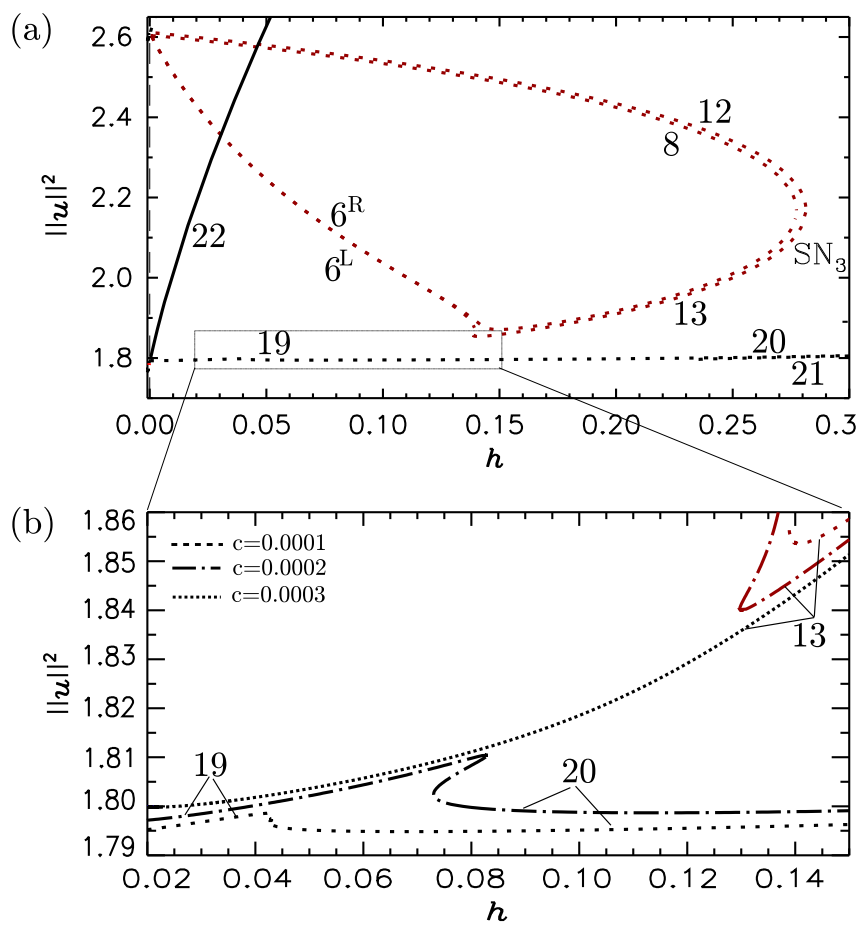

FIG. 30. Reconnection of solution branches 13 and 19. In (a) we plot a zoom of the diagram in Fig. 20, showing branches 13 and 19 for $c=0.0001$. In panel (b) a zoom of panel (a) illustrates how branches 13 and 19 approach for increasing $c$ until they reconnect for $c=0.0003$.

11, 12, 13, and 22 reconnect. In Fig. 30(a) we show a zoom of Fig. $20(c=0.0001)$ that includes the isola $\mathrm{L}_{3}$, composed by branches 8 and $6^{R}$; branches 12,13 , and $6^{L}$; and the branches 19, 20, 21, and 22. The zoom of Fig. 30(a) can be seen in Fig. 30(b) for several values of the drift strength. For $c=0.0001$, branches 19 and 20 are disconnected from 13. Increasing $c$ those sets of branches approach as shown for $c=0.0002$, and at some point they touch resulting in the reconnection of 19 with 13 for $c=0.0003$.
[1] N. Akhmediev and A. Ankiewicz (eds.), Dissipative Solitons, Lecture Notes in Physics, Vol. 661 (Springer, New York, 2005); Disipative Solitons: From Optics to Biology and Medicine, Lecture Notes in Physics, Vol. 751 (Springer, New York, 2008).

[2] J. E. Pearson, Science 261, 189 (1993); K. J. Lee et al., ibid. 261, 192 (1993).

[3] I. Müller, E. Ammelt, and H.-G. Purwins, Phys. Rev. Lett. 82, 3428 (1999).

[4] O. Thual and S. Fauve, J. Phys. (France) 49, 1829 (1988).

[5] W. A. Macfadyen, Geogr. J. 116, 199 (1950).

[6] L. A. Lugiato, IEEE J. Quantum Electron. 39 (2) (2003); M. Tlidi, P. Mandel, and R. Lefever, Phys. Rev. Lett. 73, 640 (1994); B. Schapers et al., ibid. 85, 748 (2000).

[7] S. Barland et al., Nature (London) 419, 699 (2002).

[8] P. B. Umbanhowar, F. Melo, and H. L. Swinney, Nature 382, 793 (1996)
[9] W. J. Firth, A. Lord, and A. J. Scroggie, Phys. Scr. T67, 12 (1996); W. J. Firth, G. K. Harkness, A. Lord, J. McSloy, D. Gomila, and P. Colet, J. Opt. Soc. Am. B 19, 747 (2002).

[10] V. K. Vanag and I. R. Epstein, Chaos 17, 037110 (2007).

[11] F. Leo, L. Gelens, P. Emplit, M. Haelterman, and S. Coen, Opt. Express 21, 9180 (2013).

[12] D. Michaelis, U. Peschel, C. Etrich, and F. Lederer, IEEE J. Quantum Electron. 39, 255 (2003).

[13] P. Parra-Rivas, D. Gomila, M. A. Matias, S. Coen, and L. Gelens, Phys. Rev. A 89, 043813 (2014).

[14] D. Gomila, M. A. Matias, and P. Colet, Phys. Rev. Lett. 94, 063905 (2005); D. Gomila, A. Jacobo, M. A. Matias, and P. Colet, Phys. Rev. E 75, 026217 (2007).

[15] E. M. Izhikevich, Dynamical Systems in Neuroscience: The Geometry of Excitability and Bursting (MIT Press, Cambridge, MA, 2007).

[16] A. Jacobo, D. Gomila, M. A. Matias, and P. Colet, New J. Phys. 14, 013040 (2012). 
[17] P. Parra-Rivas, D. Gomila, M. A. Matías, and P. Colet, Phys. Rev. Lett. 110, 064103 (2013).

[18] J. M. Soto-Crespo and N. Akhmediev, Phys. Rev. E 66, 066610 (2002).

[19] L. Gelens and E. Knobloch, Phys. Rev. E 84, 056203 (2011).

[20] M. Santagiustina, P. Colet, M. San Miguel, and D. Walgraef, Phys. Rev. Lett. 79, 3633 (1997).

[21] E. Louvergneaux, C. Szwaj, G. Agez, P. Glorieux, and M. Taki, Phys. Rev. Lett. 92, 043901 (2004).

[22] H. Ward, M. N. Ouarzazi, M. Taki, and P. Glorieux, Eur. Phys J. D 3, 275 (1998); M. Santagiustina, P. Colet, M. S. Miguel, and D. Walgraef, Opt. Lett. 23, 1167 (1998).

[23] B. Schapers, T. Ackemann, and W. Lange, IEEE J. Quantum Electron. 39, 227 (2003).

[24] K. L. Babcock, G. Ahlers, and D. S. Cannell, Phys. Rev. Lett. 67, 3388 (1991).

[25] B. von Haeften and G. Izús, Phys. Rev. E 67, 056207 (2003).

[26] M. Cross and P. Hohenberg, Rev. Mod. Phys. 65, 851 (1993).

[27] D. Walgraef, Spatio-temporal Pattern Formation (SpringerVerlag, New York, 1997).

[28] J. Guckenheimer and P. Holmes, Nonlinear Oscillations, Dynamical Systems, and Bifurcations of Vector Fields (Springer, New York, 1983).

[29] Y. A. Kuznetsov, Elements fo Applied Bifurcation Theory, 3rd ed. (Springer, New York, 2004).

[30] S. H. Strogatz, Nonlinear Dynamics and Chaos, 2nd ed. (Westview Press, Boulder, CO, 2014).

[31] J. Burke and E. Knobloch, Phys. Rev. E 73, 056211 (2006).

[32] P. D. Woods and A. R. Champneys, Physica D 129, 147 (1999).

[33] P. Coullet, C. Riera, and C. Tresser, Phys. Rev. Lett. 84, 3069 (2000).

[34] J. M. Soto-Crespo, N. Akhmediev, and G. Town, Opt. Commun. 199, 283 (2001).

[35] J. Burke, S. M. Houghton, and E. Knobloch, Phys. Rev. E 80, 036202 (2009).

[36] F. Takens, Publ. Math. Inst. Hautes Etud. Sci. 43, 47 (1974).
[37] R. I. Bodganov, Funct. Anal. Appl. 9, 144 (1975).

[38] S. Schecter, SIAM J. Math. Anal. 18, 1142 (1987).

[39] J. Burke and E. Knobloch, Phys. Lett. A 360, 681 (2007).

[40] P. Parra-Rivas, D. Gomila, M. A. Matias, P. Colet, and L. Gelens, Opt. Express 22, 30943 (2014).

[41] S. Coen, H. G. Randle, T. Sylvestre, and M. Erkintalo, Opt. Lett. 38, 37 (2013); S. Coen and M. Erkintalo, ibid. 38, 1790 (2013); Y. K. Chembo and C. R. Menyuk, Phys. Rev. A 87, 053852 (2013).

[42] S. Cundiff, J. Ye, and J. Hall, Sci. Am. 298, 74 (2008).

[43] P. DelHaye, A. Schliesser, O. Arcizet, T. Wilken, R. Holzwarth, and T. J. Kippenberg, Nature (London) 450, 1214 (2007).

[44] W. J. Firth and C. O. Weiss, Opt. Photonics News 13, 55 (2002)

[45] F. Pedaci, S. Barland, E. Caboche, P. Genevet, M. Giudici, J. R. Tredicce, T. Ackemann, A. Scroggie, W. Firth, G. L. Oppo, G. Tissoni, and R. Jaeger, Appl. Phys. Lett. 92, 011101 (2008).

[46] F. Leo, S. Coen, P. Kockaert, S. P. Gorza, P. Emplit, and M. Haelterman, Nat. Photon. 4, 471 (2010).

[47] V. Odent, M. Taki, and E. Louvergneaux, New J. Phys. 13, 113026 (2011).

[48] P. Parra-Rivas, D. Gomila, F. Leo, S. Coen, and L. Gelens, Opt. Lett. 39, 2971 (2014).

[49] P. Kramper, M. Kafesaki, C. M. Soukoulis, A. Birner, F. Müller, U. Güsele, R. B. Wehrspohn, J. Mlynek, and V. Sandoghdar, Opt. Lett. 29, 174 (2004).

[50] F. Pedaci, G. Tissoni, S. Barland, M. Giudici, and J. Tredicce, Appl. Phys. Lett. 93, 111104 (2008).

[51] G. Kozyreff and L. Gelens, Phys. Rev. A 84, 023819 (2011).

[52] M. Haelterman, S. Trillo, and S. Wabnitz, Opt. Commun. 91, 401 (1992).

[53] M. J. Schmidberger, D. Novoa, F. Biancalana, P. St. J. Russell, and N. Y. Joly, Opt. Express 22, 3045 (2014).

[54] E. Caboche, F. Pedaci, P. Genevet, S. Barland, M. Giudici, J. Tredicce, G. Tissoni, and L. A. Lugiato, Phys. Rev. Lett. 102, 163901 (2009). 